\title{
Modeling and simulation framework for dynamic strain localization in elasto-viscoplastic metallic materials subject to large deformations
}

\author{
H. M. Mourad ${ }^{a, *}$, C. A. Bronkhorst ${ }^{\mathrm{a}}$, V. Livescu ${ }^{\mathrm{b}}$, J. N. Plohr ${ }^{\mathrm{a}}$, E. K. Cerreta ${ }^{\mathrm{b}}$ \\ ${ }^{a}$ Theoretical Division, Los Alamos National Laboratory, Los Alamos, NM 87545, USA \\ ${ }^{b}$ Materials Science and Technology Division, Los Alamos National Laboratory, Los Alamos, NM 87545, USA
}

\begin{abstract}
This paper describes a theoretical and computational framework for the treatment of adiabatic shear band formation in rate-sensitive polycrystalline metallic materials. From a computational perspective, accurate representation of strain localization behavior has been a long-standing challenge. In addition, the underlying physical mechanisms leading to the localization of plastic deformation are still not fully understood. The proposed framework is built around an enhanced-strain finite element formulation, designed to alleviate numerical pathologies known to arise in localization problems, by allowing a localization band of given finite width (weak discontinuity) to be embedded within individual elements. The mechanical threshold strength (MTS) model is used to represent the temperature and strain rate-dependent viscoplastic response of the material. This classical flow stress model employs an internal state variable to quantify the effect of dislocation structure evolution (work hardening and recovery). In light of growing evidence suggesting that the softening effect of dynamic recrystallization may play a significant role, alongside thermal softening, in the process of shear band formation and growth, a simple dynamic recrystallization model is proposed and cast within the context of the MTS model with the aid of the aforementioned internal state variable. An initiation criterion for shear localization in rate and temperature-sensitive materials is introduced and used in the present context of high-rate loading, where material rate-dependence is pronounced and substantial temperature increases are achieved due to the dissipative nature of viscoplastic processes. In addition, explicit time integration is adopted to facilitate treatment of the dynamic problems under consideration, where strain rates in excess of $10^{4} \mathrm{~s}^{-1}$ are typically attained. Two series of experiments are conducted on AISI 316L stainless steel, employing the commonly used top-hat sample geometry and the Split-Hopkinson Pressure Bar dynamic test system. Axi-symmetric finite element simulation results are compared to cross-sectional micrographs of recovered samples and experimental load-displacement results, in order to examine the performance of the proposed framework and demonstrate its effectiveness in treating the initiation and growth of adiabatic shear banding in dynamically loaded metallic materials. These comparisons demonstrate that thermal softening alone is insufficient to induce shear localization behaviors observed in some materials, such as stainless steel, and support the hypothesis that dynamic recrystallization and/or other softening mechanisms play an essential role in this process.
\end{abstract}

Keywords: Strain localization, adiabatic shear banding, finite element method, enhanced strain methods, explicit dynamic analysis, viscoplasticity, dynamic recrystallization, split-Hopkinson pressure bar experiments

\section{Introduction}

Adiabatic shear localization is an important physical process that affects the general class of poly-crystalline metallic materials considered in the present paper. Subtractive machining processes rely upon shear localization and failure for successful high-rate removal of material, which is required in order to achieve a high-quality surface finish, to reduce tool wear and maximize time between periods of retooling, and to improve the overall energy and cost

\footnotetext{
${ }^{*}$ Corresponding author.

Email address: hmourad@lanl.gov (H. M. Mourad)
} 
efficiency of the manufacturing process. In certain defense applications, the propensity to form adiabatic shear bands is a desirable material attribute; it is designed into certain structural components to minimize the energy necessary to produce a disbursed velocity field, such that the velocity is maximized. On the other hand, forming operations of either bulk or sheet metal are usually designed to avoid localized shearing as this non-uniform deformation is believed to cause flaws in the final product, and is generally considered detrimental to its in-service performance. Also, when metallic components are designed to absorb energy due to mechanical insult, the formation of adiabatic shear bands is highly undesirable. These widely varying requirements, placed upon materials subjected to different types of dynamic loading conditions, underscore the importance of understanding the physics of adiabatic shear banding and developing computational tools that enable the proper representation of this physical process, in order to facilitate the design of engineering components for various applications.

There have been a number of reviews published on the topic of adiabatic shear localization and the difficulties associated with the numerical representation of this physical process. Notable among these is the second edition of the detailed review of Dodd and Bai, which appeared recently (2012). This book treats the subject in a broad context of several applications, and differing material classes in addition to poly-crystalline metallic materials. In the first part of the book, the authors focus on metallic materials and discuss computational representation of the shear localization process, as well as advancements in our physical understanding of processes leading up to the formation of adiabatic shear bands. Of particular interest to the present work is their discussion of the softening mechanisms involved in shear band formation, which is not limited to traditional thermal softening (increase in dislocation mobility due to temperature increase), but rather extends to additional, recently-identified mechanisms. Properly identifying such mechanisms is challenging, given the very small dimensions of shear bands, and the exceedingly short time durations characteristic of dynamic loading conditions which give rise to adiabatic shear localization. In particular, the book discusses growing evidence pointing to dynamic recrystallization as an important softening mechanism in certain types of materials. Even more recently, Dodd and Bai (2014) discuss in less detail the engineering aspects of adiabatic shear banding and its implications. In addition, the work of Meyers (1994) provides an overview of the mathematics of material stability analysis and the relevant materials physics, and Wright (2002) presents a survey of mathematical models used to represent the phenomena of adiabatic shear banding. The issue of material stability is also covered by Belytschko et al. (2013), focusing on its implications with regards to successful computational strategies. They discuss the century-old recognition that strain softening leads to material instability, accompanied by a change in character (loss of hyperbolicity) of the partial differential equations governing the behavior of rateindependent solid materials under dynamic conditions. Improved representation of localization processes is discussed in the context of regularization strategies needed to avoid non-physical, mesh-dependent solutions to these equations. This practically important issue of mesh sensitivity is part of what we will attempt to address in this work.

The higher-level references cited above do not constitute an exhaustive suggested reading list on the subject of adiabatic shear banding. However, they do provide an overview of this rich field of study where significant challenges still remain. As these references attest, it has been known for many years that temperature increase due to rapid plastic deformation and the ensuing increase in dislocation mobility play an important role in the process of adiabatic shear localization. More recent results suggest, however, that thermal softening is not the only active softening mechanism in some materials. For example, in previous work by Bronkhorst et al. (2006), where forced-shear experiments are conducted on 316L stainless steel samples, a substantial drop in the measured load versus displacement behavior is linked to the concomitant formation of adiabatic shear bands. The load-carrying capacity of the sample is also shown to be significantly over-predicted computationally when accounting only for the thermal softening mechanism.

Recent work by Rittel and co-workers (Rittel et al., 2008; Osovski et al., 2012, 2013), primarily on titanium and titanium alloys, presents metallographic mechanical response evidence to suggest that dynamic recrystallization takes place within shear bands. In the latest of these three works, the authors present a computational analysis of the dynamic shear response of titanium where they account for the mechanisms of dislocation slip, mechanical twinning, and dynamic recrystallization. For the conditions studied using this material, the authors conclude that dynamic recrystallization plays a prominent role in the shear banding process. The authors also point out that there is little direct experimental evidence to quantify the evolution of this recovery process with time, in large part due to the small size of the adiabatic shear band and the very short time durations of these strongly-driven experiments. Similarly, Medyanik et al. (2007) present an adiabatic shear localization criterion based on the premise that dynamic recrystallization plays a prominent role in addition to traditional thermal softening. Notably, in their numerical analysis of 4340 steel and OFHC copper, they use distinctly different constitutive models to represent the material response inside and 
outside an adiabatic shear band, and use a temperature-based initiation criterion for dynamic recrystallization. These authors also provide a list of additional experimental studies that support the conclusion that dynamic recrystallization takes place within the shear band (e.g. Hines and Vecchio, 1997; Xu et al., 2001). Furthermore, in their detailed metallographic analysis of 201 austenitic stainless steels deformed at high rate using the forced-shear sample design, (Wang et al., 2014a,b) present high-resolution images of the material within a shear band, clearly revealing very fine and equi-axed grains. The homologous temperature is estimated to reach 0.6 due to the imposed deformation in these experiments. The authors propose that the observed microstructures are produced by both dynamic recrystallization and rotational dynamic recrystallization. Grain sizes ranging from 30 to $80 \mathrm{~nm}$ are observed by Wang et al. (2014a), and sizes of 100-300 $\mathrm{nm}$ are reported by Wang et al. (2014b).

The forced-shear top-hat sample geometry is also used in a series of dynamic experiments performed on the 304 stainless steel alloy by Xue et al. $(2005,2008)$. In both of these studies, as-received and pre-shocked materials are examined, and clear differences are observed in the adiabatic shear band initiation and growth behavior, due to significant differences in prior history between the two materials. Shear band width dimensions in the range 7-13 $\mu \mathrm{m}$ are reported, with shear strains reaching approximately 24.3 in the most highly deformed case. Although highresolution EBSD examination is not performed, available EBSD images do suggest that a very fine grain structure may exist within the shear zone. The authors do not comment on that possibility since high-resolution techniques capable of resolving these very fine grain structures have only become available very recently.

Mechanically deformed austenitic stainless steels are known to undergo the gamma-to-alpha prime martensitic phase transformation under certain loading and environmental conditions. Hecker et al. (1982) describe the physical mechanism of this transformation in this class of materials, noting the strong coupling between the defect structure evolution during plastic deformation and the nucleation of the ferritic phase. They present results of quasi-static and dynamic experiments performed on the 304 stainless steel alloy, showing that significant volume fractions of martensitic phases can be accumulated with deformation. This process, however, is strongly dependent upon the temperature of the sample; namely, the transformation process is slower at higher temperatures, with results showing that it is almost completely suppressed above $50^{\circ} \mathrm{C}$. More recent studies (Talonen and Hanninen, 2007; Das et al., 2008; Okayasu et al., 2013) examine this martensitic phase transformation process in austenitic stainless steels using EBSD metallography, and report accumulation of the martensitic phases during plastic deformation. They also report significantly slower transformation kinetics at higher temperatures, and thus reduced martensitic phase production with increasing strain rate due to adiabatic heating. This explains the fact that martensitic phases are not observed by Xue et al. $(2005,2008)$ in their detailed metallographic examination of the shear zones in forced-shear samples, used to study adiabatic shear banding in 304 stainless steel.

As mentioned above, the process of adiabatic shear band formation and growth depends strongly on the hardening and softening mechanisms active during large deformation at high strain rates. In most materials, outside the shock loading regime (i.e. with low to moderate dislocation velocities), the process of plastic slip is adequately represented by models based on thermally assisted dislocation motion (Kocks et al., 1975). Based upon that early foundational work, several models have been developed and applied to materials with different crystallographic structure (e.g., see Follansbee and Kocks, 1988; Follansbee, 2014).

For steels in particular, Abed and Makarem (2012) compare several different constitutive models and find that the model of Abed and Voyiadjis (2005), which is based on the physics of thermally activated slip, shows superior relative performance. Abed et al. (2013) use the same model successfully to predict the dynamic response of two structural steels under simple compression and forced-shear loading, with varying initial temperatures. They illustrate the model's ability to capture the temperature sensitivity of the flow stress in these steels, via comparisons to results from simple-compression experiments. The model's ability to capture the deformation rate and temperature sensitivity of the flow stress in AL-6XN stainless steel is also examined, exhibiting very good performance with initial temperature conditions in the range $77-1000 \mathrm{~K}$ and strain rates in the range $0.001-8300 \mathrm{~s}^{-1}$. In addition, a detailed mechanistic analysis of the plastic behavior of 304, 304L, 316, and 316 stainless steel alloys, performed using the MTS flow stress model, is presented by Follansbee (2012). This analysis shows that, in order to accurately represent the plastic flow resistance of these materials up to homologous temperatures of 0.5-0.6, four independent sources of resistance to dislocation motion need to be included: an athermal term representing long-range grain boundary interaction, and three temperature-dependent terms representing alloy interstitial atoms, nitrogen, and evolution of dislocation structure, respectively. Notably, the author finds no indication of martensitic phase transformation from the parent austenite within this extensive examination of data across a range of temperatures and strain rates. 
From a computational perspective, adequate representation of the strain localization process is a non-trivial task. The practical difficulty stems from the need to resolve isolated regions of localized deformation on the order of $10 \mu \mathrm{m}$ in thickness, in a general engineering problem which may involve characteristic dimensions on the order of meters. In a finite element setting, this requires an exceedingly high mesh resolution, which is cost prohibitive. Alternatively, a multi-scale approach, such as the sub-grid formulation presented herein, can be utilized to represent physical length scales smaller than the finite element grid size. Rate-independent local constitutive models exacerbate the aforementioned difficulties, since they lead to non-physical solutions characterized by localization of deformation to a set of measure zero (a discrete surface in a three-dimensional problem), and vanishing energy dissipation.

Generally, as alluded to above, proper regularization strategies must be implemented in localization problems, at the level of the constitutive model and/or the numerical discretization scheme, in order to inject a physical length scale into the governing system of equations. Otherwise, mesh dependent computational results are obtained, and convergence with mesh refinement (even at a sub-optimal rate) cannot be guaranteed. Despite assiduous research efforts that are too many to list here, no consensus has been reached yet on a definitive solution to this long-standing problem. Regularization strategies recently used in computational studies of strain localization include strain gradient theories (e.g. Abu Al-Rub and Voyiadjis, 2006; Anand et al., 2012), and fully-coupled thermo-mechanical analysis (McVeigh and Liu, 2010; McAuliffe and Waisman, 2013, 2014) taking thermal conductivity into account.

The origins of the computational framework proposed herein can be traced back to the pioneering work of Ortiz et al. (1987), where a discontinuity in the strain field, termed a weak discontinuity, is embedded within an individual finite element using an enhanced strain interpolation scheme, once the material in that element exhibits unstable behavior. This allows a localization band to be represented by embedding the two discontinuities bordering it, each in one of two adjacent elements. Motivated by these developments, an improved small-strain formulation is developed by Belytschko et al. (1988) and generalized to finite-deformation problems by Fish and Belytschko (1988). These formulations allow the localization band to be embedded within a single element rather than two neigboring ones, breaking the link between the band width and the element size. A generalization of the original technique to dynamic problems is presented by Leroy and Ortiz (1990), and used to study the effect of material rate sensitivity and inertia on the shear banding process in two-dimensional geometries.

Another class of computational methods represent localization bands as discontinuities in the displacement field, i.e. strong discontinuities. Some of the earlier members of this class of methods rely on enforcing cohesive tractionseparation laws along boundaries between neighboring elements. In a related technique (Yang et al., 2005), cohesivezone elements are replaced by specialized strain-localization elements which allow the response of the band material to be described using the same stress-strain relations governing the behavior of the surrounding continuum elements (or potentially, using different constitutive relations). Not surprisingly, however, such methods may yield results that are dependent on the mesh alignment. This drawback motivates the development of strategies for embedding strong discontinuities within element interiors (e.g. Borja and Regueiro, 2001). In this class of methods, the added displacement jumps can be viewed as fine-scale degrees of freedom within the context of the variational multiscale method Garikipati and Hughes (2000). Recent work in this area includes a treatment of propagating discontinuities presented by Linder and Armero (2007), with extensions to the finite-deformation regime (Armero and Linder, 2008), dynamic loading conditions (Armero and Linder, 2009) and three dimensional problems (Armero and Kim, 2012). Another recent development in this area is the finite-thickness band method presented by Huespe et al. (2009), and extended to the finite-deformation case by Huespe et al. (2012). A notable feature of these formulations is their ability to transition from a weak-discontinuity to a strong-discontinuity representation, making them suitable for the treatment of ductile damage and failure within a unified framework.

Other finite element methodologies rely on the introduction of additional nodal degrees of freedom, for example within the context of the extended finite element method (Song et al., 2006), to represent strong discontinuities. The main disadvantage of this approach is that the additional degrees of freedom are global, leading to an increase in the size of the problem. By contrast, embedded (weak or strong) discontinuity methods add local degrees of freedom, which can be eliminated at the element level via static condensation. For a literature survey and taxonomy of relevant computational approaches, see Jirásek (2000); Oliver et al. (2006); Borja (2008); Wu et al. (2015). Other computational approaches used in shear localization problems include the meshfree Galerkin method (Li et al., 2001, 2002), and isogeometric analysis Berger-Vergiat et al. (2014).

Detecting the onset of localization behavior in the proposed computational framework is accomplished with the aid of a material stability analysis. A linear perturbation analysis method (Anand et al., 1987; Plohr and Plohr, 2016) 
is employed, since the traditional eigenvalue analysis of the acoustic tensor is not applicable to the rate-sensitive materials being considered here (see also Arriaga et al., 2015, 2016, for alternative localization criteria). When the onset of localization behavior is detected at a certain quadrature point, the orientation of the nascent localization band is given by the material stability analysis, and the sub-grid method is immediately activated at that point. Henceforth, traction continuity conditions are enforced along the boundary between the embedded band and the surrounding matrix material, and rotation of the band under finite deformation is taken into account. The method allows the band and matrix materials to be represented by different constitutive models, and allows the thickness of the embedded band to evolve according to any appropriate model. The development of a physically realistic shear band thickness evolution model is a non-trivial undertaking, and will be the subject of future research. In the present study, we treat the shear band width as a constant material parameter, and set its value in accordance with experimental observations (e.g. Xue and Gray III, 2006).

The current paper presents a framework for the treatment of adiabatic shear banding process in poly-crystalline metallic materials, with a focus on AISI 316L stainless steel. An outline of the constitutive model used to represent the material's elasto-viscoplastic response under dynamic loading conditions is presented in Sec. 2. This includes the kinematic description and models for both slip based hardening and softening, along with a simple model of dynamic recrystallization. The foundation of the shear band nucleation criteria used with rate-dependent material descriptions is then discussed. The computational approach is described in detail in Sec. 3, and the experiments performed on the 316L material are described in Sec. 4. An analysis of experimental and computational results is presented in Sec. 5 together with a detailed discussion, followed by a brief summary and concluding remarks in Sec. 6 .

\section{Theory and constitutive modeling}

\subsection{Kinematics and governing equations}

We consider a continuum subjected to the deformation $\varphi$, over a time interval of interest, $\mathcal{T}$. Every particle $X$ in the initial configuration $\Omega_{0}$ of the continuum is mapped to a corresponding point $\boldsymbol{x}=\boldsymbol{\varphi}(X, t)$ in the deformed configuration $\Omega$ at time $t \in \mathcal{T}$. The deformation gradient tensor is defined as

$$
\mathbf{F}:=\frac{\partial \varphi}{\partial X} .
$$

In addition, the rate of deformation tensor, $\mathbf{d}$, and the spin tensor, $\mathbf{w}$, are defined by

$$
\begin{aligned}
\mathbf{d} & :=\frac{1}{2}\left(\mathbf{l}+\mathbf{l}^{\mathrm{T}}\right), \\
\mathbf{w} & :=\frac{1}{2}\left(\mathbf{l}-\mathbf{l}^{\mathrm{T}}\right),
\end{aligned}
$$

in terms of the velocity gradient

$$
\begin{aligned}
\mathbf{l} & :=\boldsymbol{\nabla} \dot{\boldsymbol{u}} \\
& =\dot{\mathbf{F}} \mathbf{F}^{-1},
\end{aligned}
$$

where $\boldsymbol{u}(\boldsymbol{x}, t):=\boldsymbol{x}-\boldsymbol{\varphi}^{-1}(\boldsymbol{x}, t)$ is the displacement field, and $\boldsymbol{\nabla}$ is the spatial gradient operator. Superposed dots designate material time derivatives. The problem is governed by the principle of conservation of linear momentum,

$$
\boldsymbol{\nabla} \cdot \boldsymbol{\sigma}=\rho \ddot{\boldsymbol{u}} \quad \text { in } \Omega,
$$

where body forces are neglected, $\sigma$ is the Cauchy stress tensor and $\rho$ is the material's mass density. The boundary of $\Omega$ is denoted by $\Gamma$ and is partitioned into disjoint complementary subsets $\Gamma_{u}$ and $\Gamma_{h}$, where essential and natural boundary conditions are applied, respectively:

$$
\begin{aligned}
\boldsymbol{u} & =\overline{\boldsymbol{u}} & & \text { on } \Gamma_{u}, \\
\boldsymbol{\sigma} \cdot \boldsymbol{n} & =\overline{\boldsymbol{h}} & & \text { on } \Gamma_{h} .
\end{aligned}
$$


Here, $\overline{\boldsymbol{u}}$ and $\overline{\boldsymbol{h}}$ are given functions of $\boldsymbol{x}$ and $t$, and $\boldsymbol{n}$ denotes the outward unit vector normal to $\Gamma_{h}$. Initial conditions are expressed as follows:

$$
\begin{array}{ll}
\boldsymbol{u}(\boldsymbol{x}, 0)=\boldsymbol{u}_{0}(\boldsymbol{x}) & \forall \boldsymbol{x} \in \Omega, \\
\dot{\boldsymbol{u}}(\boldsymbol{x}, 0)=\dot{\boldsymbol{u}}_{0}(\boldsymbol{x}) & \forall \boldsymbol{x} \in \Omega,
\end{array}
$$

where the functions $\boldsymbol{u}_{0}$ and $\dot{\boldsymbol{u}}_{0}$ are given.

\subsection{Corotational formulation of elasto-viscoplasticity}

We adopt a rotation-neutralized description of the constitutive equations of hypoelastic-viscoplasticity, arrived at with the aid of a local coordinate system which rotates with the material (see Mourad et al., 2014, and references therein). In this framework, the rotation of the neighborhood of a material point is characterized by the orthogonal tensor $\boldsymbol{\Lambda}$, which is subject to the following evolution equation

$$
\dot{\Lambda}=\mathbf{w} \mathbf{\Lambda},
$$

with initial condition $\mathbf{\Lambda}=\mathbb{1}$, corresponding to the initial (undeformed) state. Here, $\mathbf{w}$ is the spin tensor, and $\mathbb{1}$ denotes the second-order identity tensor. Accordingly, the corotational description, $\mathbf{D}$, of the rate of deformation tensor is obtained from its spatial description, $\mathbf{d}$, via

$$
\mathbf{D}=\boldsymbol{\Lambda}^{\mathrm{T}} \mathbf{d} \boldsymbol{\Lambda} .
$$

In metallic materials of interest, elastic strains are expected to remain small. With this notion in mind, the rate of deformation tensor is decomposed additively, into elastic and viscoplastic components $\mathbf{D}^{\mathrm{e}}$ and $\mathbf{D}^{\mathrm{p}}$, respectively:

$$
\mathbf{D}=\mathbf{D}^{\mathrm{e}}+\mathbf{D}^{\mathrm{p}} .
$$

Rate constitutive equations of the form

$$
\dot{\boldsymbol{\Sigma}}=\mathbb{C}^{\mathrm{e}}: \mathbf{D}^{\mathrm{e}},
$$

are used to characterize the stress response of the material. Here, $\boldsymbol{\Sigma}$ denotes the corotational representation of the Cauchy stress tensor, given in terms of its spatial representation, $\sigma$, by the transformation

$$
\boldsymbol{\Sigma}=\boldsymbol{\Lambda}^{\mathrm{T}} \boldsymbol{\sigma} \boldsymbol{\Lambda} .
$$

Differentiating this expression with respect to time reveals the relationship

$$
\dot{\boldsymbol{\Sigma}}=\boldsymbol{\Lambda}^{\mathrm{T}} \stackrel{\nabla}{\boldsymbol{\sigma}} \boldsymbol{\Lambda}
$$

where $\stackrel{\nabla}{\sigma}:=\dot{\sigma}+\sigma \mathbf{w}-\mathbf{w} \sigma$ is the Jaumann-Zaremba objective stress rate. In the present work, we restrict our attention to isotropic hypoelastic response, i.e. the fourth-order elasticity tensor is given by

$$
\mathbb{C}^{\mathrm{e}}=K \mathbb{1} \otimes \mathbb{1}+2 G\left(\mathbb{I}-\frac{1}{3} \mathbb{1} \otimes \mathbb{1}\right),
$$

where the constants $K$ and $G$ are the elastic bulk and shear moduli, respectively, and II is the fourth-order symmetric identity tensor. The Mises yield criterion is adopted, and admissible states of stress are required to lie on or within the yield surface, as in the case of rate-independent plasticity:

$$
\mathcal{F}:=\|\mathbf{T}\|-R \leq 0,
$$

where $\mathbf{T}:=\boldsymbol{\Sigma}-\frac{1}{3} \operatorname{trace}(\boldsymbol{\Sigma}) \mathbb{1}$ is the deviatoric part of the corotational stress, and $\|\bullet\|:=\sqrt{\bullet: \bullet}$ denotes the Eulerian norm. Isotropic hardening/softening are incorporated, respectively, via expansion/contraction of the yield surface, as measured by its radius

$$
R:=\sqrt{\frac{2}{3}} \hat{\sigma},
$$


defined here in terms of the flow stress, $\hat{\sigma}$, given by (24). Associated flow is assumed:

$$
\mathbf{D}^{\mathrm{p}}=\dot{\lambda} \frac{\partial \mathcal{F}}{\partial \mathbf{\Sigma}}=\dot{\lambda} \mathbf{N}
$$

where $\lambda$ is the viscoplastic multiplier, and

$$
\mathbf{N}:=\frac{\mathbf{T}}{\|\mathbf{T}\|},
$$

is the unit normal to the yield surface. Thus, we have the following expressions for the equivalent viscoplastic strain rate,

$$
\dot{\bar{\varepsilon}}^{\mathrm{p}}:=\sqrt{\frac{2}{3} \mathbf{D}^{\mathrm{p}}: \mathbf{D}^{\mathrm{p}}}=\sqrt{\frac{2}{3}} \dot{\lambda}
$$

and the accumulated viscoplastic strain,

$$
\bar{\varepsilon}^{\mathrm{p}}:=\int_{0}^{t} \dot{\bar{\varepsilon}}^{\mathrm{p}} \mathrm{d} t=\sqrt{\frac{2}{3}} \lambda
$$

\subsection{The MTS flow-stress model}

The mechanical threshold stress (MTS) model (Follansbee and Kocks, 1988; Follansbee et al., 1990; Mourad et al., 2014) can be regarded as a complex, nonlinear isotropic hardening law, which accounts for strain- and strain-rate hardening, as well as thermal softening effects. It considers the combined effect of different types of barriers to dislocation glide, in order to characterize the material's resistance to inelastic deformation. This resistance is separated into an athermal component, representing the interactions of dislocations with long-range barriers such as grain boundaries, and thermal components representing dislocation interactions with short-range obstacles which can be overcome via thermal activation, e.g. forest dislocations. The MTS is the resistance to inelastic deformation at zero absolute temperature. Adjusting this resistance to the actual conditions of temperature, $\theta$, and strain rate, $\dot{\bar{\varepsilon}}^{\mathrm{p}}$, gives the flow stress, $\hat{\sigma}$, of the material:

$$
\hat{\sigma}=\hat{\sigma}_{a}+\frac{\mu(\theta)}{\mu_{0}}\left[S_{i}\left(\theta, \dot{\bar{\varepsilon}}^{\mathrm{p}}\right) \hat{\sigma}_{i}+S_{\epsilon}\left(\theta, \dot{\bar{\varepsilon}}^{\mathrm{p}}\right) \hat{\sigma}_{\epsilon}\right] .
$$

Here, the constant athermal component of the flow stress is denoted by $\hat{\sigma}_{a}$, while its thermal components are represented by the remaining term on the right side of Eq. (24). More specifically, $\hat{\sigma}_{i}$ is the constant intrinsic lattice resistance at $\theta=0 \mathrm{~K}$ and $\hat{\sigma}_{\epsilon}$ represents the non-constant flow resistance at $\theta=0 \mathrm{~K}$ due to evolving dislocation structure. Rate and temperature kinetics are introduced via the pre-multiplying terms $S_{i}$ and $S_{\epsilon}$. The temperaturedependent shear modulus is denoted by $\mu$, and $\mu_{0}$ is its value at absolute zero temperature. The shear modulus is given as a function of temperature by the following empirical expression:

$$
\mu(\theta)=\mu_{0}-\frac{D_{0}}{\exp \left(\frac{\theta_{0}}{\theta}\right)-1},
$$

where $\theta_{0}$ is a reference temperature. The pre-multiplying term $S_{i}$, appearing in Eq. (24), is given by

$$
S_{i}\left(\theta, \dot{\bar{\varepsilon}}^{\mathrm{p}}\right)=\left(1-\left[\frac{k \theta}{\mu b^{3} g_{0 i}} \ln \left(\frac{\dot{\varepsilon}_{0 i}}{\dot{\bar{\varepsilon}}^{\mathrm{p}}}\right)\right]^{\frac{1}{q_{i}}}\right)^{\frac{1}{p_{i}}},
$$

where $k$ is Boltzmann's constant, $b$ is the magnitude of the Burgers vector, $g_{0 i}$ is a normalized activation energy, $\dot{\varepsilon}_{0 i}$ is a reference strain rate, $p_{i} \in[0,1]$ and $q_{i} \in[1,2]$ are exponents which determine the shape of the energy barrier profile (Kocks et al., 1975). A similar expression gives the pre-multiplying term $S_{\epsilon}$ :

$$
S_{\epsilon}\left(\theta, \dot{\bar{\varepsilon}}^{\mathrm{p}}\right)=\left(1-\left[\frac{k \theta}{\mu b^{3} g_{0 \epsilon}} \ln \left(\frac{\dot{\varepsilon}_{0 \epsilon}}{\dot{\bar{\varepsilon}}^{\mathrm{p}}}\right)\right]^{\frac{1}{q_{\epsilon}}}\right)^{\frac{1}{p_{\epsilon}}} .
$$

Reflecting the evolution of the dislocation structure, the mechanical threshold $\hat{\sigma}_{\epsilon}$ evolves as

$$
\frac{\mathrm{d} \hat{\sigma}_{\epsilon}}{\mathrm{d} \bar{\varepsilon}^{\mathrm{p}}}=h_{0}\left(1-\frac{\hat{\sigma}_{\epsilon}}{\hat{\sigma}_{\epsilon s}}\right)^{\kappa},
$$


where

$$
h_{0}=A_{0}+A_{1} \log \left(\dot{\bar{\varepsilon}}^{\mathrm{p}}\right)+A_{2} \sqrt{\dot{\bar{\varepsilon}}^{\mathrm{p}}}-A_{3} \theta+A_{4} \theta^{-A_{5}},
$$

and $\hat{\sigma}_{\epsilon S}$ is the saturation threshold stress given by:

$$
\hat{\sigma}_{\epsilon s}=\hat{\sigma}_{\epsilon s 0}\left(\frac{\dot{\bar{\varepsilon}}^{\mathrm{p}}}{\dot{\varepsilon}_{0 \epsilon s}}\right)^{\frac{k \theta}{\mu b^{3} B_{0 \epsilon s}}},
$$

where $g_{0 \epsilon s}$ is a normalized activation energy and $\dot{\varepsilon}_{0 \epsilon s}$ is a reference strain rate.

\subsection{Temperature evolution}

When the strain rate exceeds $1 \mathrm{~s}^{-1}$ at any material point, inside or outside shear band regions, adiabatic conditions are assumed to hold locally, i.e. the rate of heat conduction is considered negligible compared to that of local heat generation due to viscoplastic work. Thus, the rate of temperature increase at such a material point is given by

$$
\dot{\theta}=\frac{\Psi}{\rho C_{p}} \wp,
$$

up to the melting point, $\theta=\theta_{\text {melt }}$, in terms of the local viscoplastic power, $\wp$, defined as:

$$
\wp:=\boldsymbol{\Sigma}: \mathbf{D}^{\mathrm{p}}=\|\mathbf{T}\| \dot{\lambda} .
$$

In Eq. (31), $\Psi$ denotes the fraction of $\wp$ that is converted to heat, $\rho$ is the mass density, and

$$
C_{p}=C_{0}+C_{1} \theta+C_{2} \theta^{-2}
$$

is the specific heat capacity of the material. Conversely, when the material deforms at a strain rate of $1 \mathrm{~s}^{-1}$ or less, isothermal conditions $(\dot{\theta}=0)$ are assumed. Bronkhorst et al. (2006) have shown the above assumptions to be valid under the loading conditions considered in the present work.

\subsection{Dynamic recrystallization}

Prior work on the 316L stainless steel alloy (Bronkhorst et al., 2006) suggested that the softening mechanisms accounted for by the MTS model were insufficient to reproduce the force-displacement response observed experimentally during the formation and growth of adiabatic shear bands. Recent work on the study of alloy steels has suggested that the process of dynamic recrystallization is active when high temperature conditions are developed during conditions of large plastic deformation within the shear zone. Prior calculations have suggested that temperatures approaching that of melting are developed in these materials (Bronkhorst et al., 2006). Although still a current area of research, the representation of mechanical behavior of material within the region of extreme localization is important for our ability to predict the deformation state of that material as well as the state of the material at which point failure occurs. It is certainly well accepted that thermal softening plays an important role in the process of adiabatic shear band formation. Recent work has suggested that dynamic recrystallization could be a contributing factor to the softening response of the material (Rittel et al., 2008; Osovski et al., 2013; Wang et al., 2014a,b) under adiabatic loading conditions.

There has been substantial work in the area of dynamic recrystallization modeling. One approach is based on considering the nucleation and subsequent growth of recrystallized grains. Since our ability to model the nucleation process is limited (Lin and Chen, 2011), we assume that recrystallization begins when the local temperature reaches a threshold value, $\theta_{\mathrm{drx}}$. Note that although dynamic recrystallization contributes to softening, which in turn leads to shear localization, an independent initiation criterion is used for localization (Sec. 2.6). Recrystallized grains are assumed to grow at the expense of their neighbors via thermally activated grain boundary migration. Therefore, their growth rate is expressed as follows (see Davies, 1997; Mourad and Garikipati, 2006, and references therein for a more detailed discussion):

$$
\dot{u}_{\mathrm{drx}}=\left[\frac{b^{2}}{k \theta} D_{\mathrm{drx}} \exp \left(\frac{-Q}{R \theta}\right)\right] p_{\mathrm{drx}},
$$


where the Arrhenius term in square brackets represents the grain boundary mobility, $D_{\mathrm{drx}}$ is a diffusion coefficient, $Q$ is the activation energy for grain boundary migration, $R$ is the universal gas constant, and $p_{\mathrm{drx}}$ is the driving force acting on a unit area of the grain boundary. This thermodynamic driving force is due to the difference in strain energy density between the dislocation-free recrystallized grains and their strain-hardened neighbors (Porter and Easterling, 1992). Thus, growth of the recrystallized grains under the effect of $p_{\mathrm{drx}}$ leads to a reduction in total free energy of the poly-crystalline system. The driving force is also related to the resistance to plastic deformation due to the evolved dislocation state in strain-hardened material (Rittel et al., 2006). Noting that dislocation content, and associated strain energy, stored in the material as a result of strain hardening are quantified in the context of the MTS model by $\hat{\sigma}_{\epsilon}$, we posit

$$
p_{\mathrm{drx}}=\hat{\sigma}_{\epsilon}^{\mathrm{init}},
$$

where $\hat{\sigma}_{\epsilon}^{\text {init }}$ is the value of $\hat{\sigma}_{\epsilon}$ at the time of initiation of the recrystallization process, $t=t_{\mathrm{drx}}$.

Since recrystallization affects the dislocation structure in the material, the flow resistance is given by

$$
\hat{\sigma}=\hat{\sigma}_{a}+\frac{\mu(\theta)}{\mu_{0}}\left[S_{i}\left(\theta, \dot{\bar{\varepsilon}}^{\mathrm{p}}\right) \hat{\sigma}_{i}+S_{\epsilon}\left(\theta, \dot{\bar{\varepsilon}}^{\mathrm{p}}\right) \hat{\sigma}_{\epsilon}^{\mathrm{drx}}\right],
$$

after the onset of dynamic recrystallization; cf. Eq. (24). Here, for simplicity, strain hardening mechanisms are ignored during and after the recrystallization process, and hence

$$
\hat{\sigma}_{\epsilon}^{\mathrm{drx}}:=f(\theta, t) \hat{\sigma}_{\epsilon}^{\mathrm{init}},
$$

where

$$
f(\theta, t):=1-\frac{u_{\mathrm{drx}}^{3}}{d^{3}},
$$

is the volume fraction of material which has not yet undergone dynamic recrystallization, and $d$ is the recrystallized grain size. Noting that at the beginning of the recrystallization process $\left(u_{\mathrm{drx}}=0\right)$, we have $\hat{\sigma}_{\epsilon}^{\mathrm{drx}}=\hat{\sigma}_{\epsilon}^{\text {init }}$, and at the conclusion of the process $\left(u_{\mathrm{drx}}=d\right)$, we have $\hat{\sigma}_{\epsilon}^{\mathrm{drx}}=0$ which is indicative of a material in its fully annealed state, it can be seen that Eq. (34) forms the basis for the rate of softening due to dynamic recrystallization.

\subsection{Initiation of shear localization}

The onset of strain localization due to the competing effects of hardening and softening mechanisms in ratesensitive materials has been the subject of several studies in the past (e.g. Wright and Batra, 1985; Anand et al., 1987; Wright, 2002; Ling and Belytschko, 2009). More recent work by Plohr and Plohr (2016) draws upon these developments and proposes a criterion based on $J_{2}$ flow theory, employing the PTW model (Preston et al., 2003) for the ratedependent flow stress of metallic poly-crystalline materials, and adapts the linear perturbation analysis of Anand et al. (1987) for use with the specific expressions representing the rate sensitive response of the material. Here, we adapt the criterion and expressions presented by Plohr and Plohr (2016) to the conditions of thermal softening and dynamic recrystallization presented above.

We consider the deformation of a viscoplastic material in simple shear, governed by the equations of balance of momentum (6) and energy (31), together with Eqs. (24) and (36) for the flow stress of the material, respectively, before and after the onset of dynamic recrystallization. We denote by $\boldsymbol{y}_{*}$ a solution, composed of homogeneous velocity, stress and temperature fields, that satisfies this system of governing equations. To assess the stability of this homogeneous solution, we consider perturbed solutions of the form

$$
\tilde{\boldsymbol{y}}=\boldsymbol{y}_{*} \exp \left(i \xi x_{n}\right) \exp (\eta t),
$$

i.e. we assume the perturbations to be periodic in space in the direction $\overline{\boldsymbol{n}}$ with wave number $\xi$, and denote by $x_{n}$ the distance along $\overline{\boldsymbol{n}}$. In order for the homogeneous solution to become unstable, a perturbed solution with a real, positive time rate of growth must exist, i.e.

$$
\eta_{\max }:=\max _{\xi, \overline{\boldsymbol{n}}} \eta>0 .
$$

Substituting the perturbed solution $\tilde{y}$ into the system of governing equations, and writing it in matrix form

$$
\mathrm{A} y_{*}=0,
$$


we note that non-trivial homogeneous solutions, $\boldsymbol{y}_{*} \neq \mathbf{0}$, require

$$
\operatorname{det} \mathbf{A}=0,
$$

which leads to a characteristic equation of the form $C_{0} \eta^{2}+C_{1} \eta+C_{2}=0$, under dynamic, adiabatic conditions. Expressions for the coefficients of this equation, given by Anand et al. (1987), show that both $C_{0}$ and $C_{1}$ are positive in the class of pressure-insensitive materials of interest here, and thus the growth rate can be positive only if $C_{2}<0$, or equivalently, if

$$
\frac{-\Psi}{\rho C_{p}} \frac{\partial \hat{\sigma}}{\partial \theta}>\frac{1}{\hat{\sigma}} \frac{\partial \hat{\sigma}}{\partial \bar{\varepsilon}^{\mathrm{p}}}
$$

In other words, the homogeneous deformation mode becomes unstable only if the effect of thermal softening is more pronounced than that of strain hardening. It is noted that linearization of the flow stress expressions (24) and (36) is required in order to evaluate the partial derivatives appearing in the above condition which, remarkably, is independent of the wave number and the rate dependence of the material response.

In the present work, we assume that shear bands oriented normal to $\bar{n}$ develop once condition (43) is met, although this condition is necessary but not sufficient. As Arriaga et al. (2016) point out, the analysis described above is based on the spectral analysis of the operator A in Eq. (42) and hinges on the notion that the perturbation mode with the largest positive exponential growth rate will eventually dominate the solution, which presupposes a constant or very slowly changing modal spectrum. However, this may not be the case especially in localization problems where instantaneous response is not necessarily indicative of long-term behavior. Arriaga et al. (2016) propose a generalized stability analysis that is more adequate for this class of problems and more efficient from a computational standpoint.

\section{Computational methods}

\subsection{Conventional finite element formulation}

In order to arrive at the weak form of the problem, we begin by multiplying the governing equation (6) by a test function $\boldsymbol{w}(\boldsymbol{x})$ and integrating their product over the current configuration $\Omega$. Making use of the divergence theorem in this procedure, and invoking the boundary conditions (7)-(8), we obtain the integral equation

$$
\int_{\Omega} \rho \boldsymbol{w} \cdot \ddot{\boldsymbol{u}} \mathrm{d} \Omega+\int_{\Omega} \nabla \boldsymbol{w}: \sigma \mathrm{d} \Omega=\int_{\Gamma_{h}} \boldsymbol{w} \cdot \overline{\boldsymbol{h}} \mathrm{d} \Gamma,
$$

for which we seek a solution $\boldsymbol{u}$ in the space of admissible trial functions,

$$
\mathscr{U}:=\left\{\boldsymbol{u} \mid u_{i} \in H^{1}(\Omega), \boldsymbol{u}=\overline{\boldsymbol{u}} \text { on } \Gamma_{u}\right\} .
$$

Here, $u_{i}$ represents an individual component of the vector $\boldsymbol{u}$. Denoting by $L_{2}(\Omega)$ the space of square-integrable functions defined on $\Omega$, the Sobolev space $H^{1}(\Omega)$ is defined as the set of functions in $L_{2}(\Omega)$ with first-order spatial derivatives also belonging to $L_{2}(\Omega)$. The solution is required to satisfy Eq. (44) for all test functions $\boldsymbol{w}$ belonging to the space

$$
\mathscr{V}:=\left\{\boldsymbol{w} \mid w_{i} \in H^{1}(\Omega), \boldsymbol{w}=\mathbf{0} \text { on } \Gamma_{u}\right\} .
$$

The deformed configuration $\Omega$ is partitioned into $n_{\mathrm{e}}$ disjoint element domains $\Omega^{e}$ with boundaries $\Gamma^{e}$, forming the spatial discretization $\Omega^{h}=\bigcup_{e=1}^{n_{\mathrm{e}}} \Omega^{e}$. In addition, the trial and test functions are approximated by their finitedimensional counterparts, defined as follows:

$$
\begin{aligned}
& \boldsymbol{u}^{h}:=\mathbf{N ~ d}, \\
& \boldsymbol{w}^{h}:=\mathbf{N} \mathbf{c},
\end{aligned}
$$

where $\mathbf{N}(\boldsymbol{x})$ is a matrix of locally supported nodal basis functions, $\mathbf{d}(t)$ is the vector of nodal degrees of freedom, and $\mathbf{C}$ is a vector of arbitrary nodal constants. We also introduce the array $\mathbf{B}$, whose elements are the spatial gradients of the basis functions, such that

$$
\boldsymbol{\nabla} \boldsymbol{w}^{h}=\mathbf{B c},
$$


or equivalently in indicial notation (with summation implied on repeated indices here and henceforth, unless explicitly stated otherwise),

$$
\frac{\partial w_{i}^{h}}{\partial x_{j}}=\mathrm{B}_{i j A} \mathrm{C}_{A} .
$$

Thus, Eq. (44) is written in the following semi-discrete matrix form

$$
\mathbf{M} \ddot{d}+\mathbf{f}^{\text {int }}=\mathbf{f}^{\text {ext }},
$$

where $\mathbf{M}$ is the mass matrix, $\mathbf{f}^{\text {int }}$ is the internal force vector, and $\mathbf{f}^{\text {ext }}$ is the external force vector, whose respective elements are given by

$$
\begin{aligned}
\mathrm{M}_{A B} & =\sum_{e=1}^{n_{\mathrm{e}}} \int_{\Omega^{e}} \rho \mathrm{N}_{i A} \mathrm{~N}_{i B} \mathrm{~d} \Omega, \\
\mathrm{f}_{B}^{\text {ext }} & =\sum_{e=1}^{n_{\mathrm{e}}} \int_{\Gamma_{h}^{e}} \mathrm{~N}_{i B} \bar{h}_{i} \mathrm{~d} \Gamma, \\
\mathrm{f}_{B}^{\text {int }} & =\sum_{e=1}^{n_{\mathrm{e}}} \int_{\Omega^{e}} \mathrm{~B}_{i j B} \sigma_{i j} \mathrm{~d} \Omega .
\end{aligned}
$$

Here $\Gamma_{h}^{e}$ is the subset of $\Gamma^{e}$ on which natural boundary conditions are prescribed.

An axi-symmetric formulation of the above equations is employed in the present work, exploiting symmetry of the domain geometry and boundary conditions, as well as isotropy of the material response in the problems under consideration. This formulation is implemented in the explicit branch of the finite element code ABAQUS (2014), in the form of a user-defined four-noded quadrilateral element, with two degrees of freedom per node (radial and axial displacement components). In this implementation, we make use of generalized selective integration techniques in order to alleviate pathological mesh locking behaviors. The use of such mitigation strategies is of vital importance in the problems under consideration, where the material response is expected to be nearly incompressible due to extensive isochoric plastic flow. For this purpose, in practice, Eq. (54) is replaced by the following definition of the internal force vector

$$
\mathrm{f}_{B}^{\mathrm{int}}=\sum_{e=1}^{n_{\mathrm{e}}} \int_{\Omega^{e}} \overline{\mathrm{B}}_{i j B} \sigma_{i j} \mathrm{~d} \Omega .
$$

in terms of the $\overline{\mathbf{B}}$ operator defined in Appendix A. Despite the use of this B-bar technique, the term "conventional" is used here loosely to describe the current formulation, simply to distinguish it from the sub-grid formulation of Sec. 3.2.

\subsection{Sub-grid finite element formulation}

Accurate representation of the strain localization process using conventional finite element techniques, such as the ones described in Sec. 3.1, requires shear bands to be resolved explicitly, which can be prohibitively expensive, given the exceedingly small width of a shear band. In order to circumvent this difficulty, we develop a sub-grid finite element formulation based on the embedded-zone element of Fish and Belytschko (1988), which allows each shear band segment to be embedded within an individual finite element. The formulation is based on a generalization of the $\mathrm{Hu}-$ Washizu variational principle to dynamic problems. Treating the displacement $\boldsymbol{u}$, velocity gradient $\mathbf{l}$, and stress $\boldsymbol{\sigma}$ as independent field variables, the three-field weak form can be expressed as follows,

$$
\int_{\Omega} \boldsymbol{w} \cdot(\rho \ddot{\boldsymbol{u}}-\boldsymbol{\nabla} \cdot \boldsymbol{\sigma}) \mathrm{d} \Omega-\int_{\Omega} \mathbf{k}:\left(\boldsymbol{\sigma}-\boldsymbol{\Lambda} \boldsymbol{\Sigma} \boldsymbol{\Lambda}^{\mathrm{T}}\right) \mathrm{d} \Omega-\int_{\Omega} \boldsymbol{\tau}:(\mathbf{l}-\boldsymbol{\nabla} \dot{\boldsymbol{u}}) \mathrm{d} \Omega-\int_{\Gamma_{h}} \boldsymbol{w} \cdot(\overline{\boldsymbol{h}}-\boldsymbol{\sigma} \cdot \boldsymbol{n}) \mathrm{d} \Gamma=0,
$$

revealing the corresponding Euler-Lagrange equations, cf. Eqs. (4), (6), (8) and (15). Admissible solutions in this case, $(\boldsymbol{u}, \mathbf{l}, \boldsymbol{\sigma}) \in \mathscr{U} \times \mathscr{L} \times \mathscr{S}$, must satisfy Eq. (56) for arbitrary variations $(\boldsymbol{w}, \mathbf{k}, \tau) \in \mathscr{V} \times \mathscr{L} \times \mathscr{S}$, where $\mathscr{V}$ is defined by (46), and

$$
\begin{aligned}
& \mathscr{L}:=\left\{\mathbf{k} \mid \mathrm{k}_{i j} \in L_{2}(\Omega)\right\}, \\
& \mathscr{S}:=\left\{\boldsymbol{\tau} \mid \tau_{i j} \in L_{2}(\Omega), \tau=\tau^{\mathrm{T}}\right\} .
\end{aligned}
$$


The formulation is simplified significantly by introducing the approximation

$$
\mathbf{l}^{h}=\tilde{\mathbf{B}} \dot{\mathbf{d}}
$$

along with a discrete approximation for the stress that is orthogonal to $\left(\mathbf{l}^{h}-\boldsymbol{\nabla} \dot{\boldsymbol{u}}^{h}\right)$; see Simo and Hughes (1986) for details. With this setup, we recover the semi-discrete weak form of the conventional method, given by Eqs. (51)-(53) and the following expression for the internal force vector in lieu of (55):

$$
\mathrm{f}_{B}^{\mathrm{int}}=\sum_{e=1}^{n_{\mathrm{e}}} \int_{\Omega^{e}} \tilde{\mathrm{B}}_{i j B}\left(\Lambda_{i p} \Sigma_{p q} \Lambda_{j q}\right) \mathrm{d} \Omega .
$$

Remarkably, compared to the conventional formulation of Sec. 3.1, the only change involves using the assumed velocity gradient $\mathbf{l}^{h}$ instead of $\boldsymbol{\nabla} \dot{\boldsymbol{u}}^{h}$ (and accordingly, the $\tilde{\mathbf{B}}$ operator instead of $\overline{\mathbf{B}}$ ) to compute the internal force vector.

Localization processes are characterized by steep gradients (modeled here as discontinuities) in strain and/or strain-rate fields. Conventional finite element methods are capable of representing such discontinuities at inter-element boundaries, but not within individual elements. The present sub-grid method overcomes this shortcoming by embedding a localization band of given width $\omega$ within an element of size $h \geqslant \omega$, thus injecting a physical length scale into the finite element equations, and obviating the need for excessive mesh refinement by improving accuracy when $h$ is large relative to $\omega$. This is achieved through the use of a $\tilde{\mathbf{B}}$ operator defined as follows:

$$
\tilde{\mathrm{B}}_{i j A}:=\left(\delta_{i k} \delta_{j l}+\alpha \mathrm{H}_{i j} \mathrm{H}_{k l}\right) \overline{\mathrm{B}}_{k l A},
$$

where $\delta_{i j}$ is the Kronecker delta and $\alpha$ is a scalar parameter which assumes the value $\alpha=\alpha^{b}$ in the localization band, and $\alpha=-\alpha^{m}$ in the matrix material surrounding the band, thereby rendering the velocity gradient field discontinuous. The relation

$$
\alpha^{b}=\left(\frac{h}{\omega}-1\right) \alpha^{m}
$$

is implied by the following compatibility condition (see Fish and Belytschko, 1988, for details):

$$
\int_{\Omega}\left(\mathbf{l}^{h}-\nabla \dot{\boldsymbol{u}}^{h}\right) \mathrm{d} \Omega=0 .
$$

The tensor $\mathbf{H}$ is defined in the following manner,

$$
\mathbf{H}:=\overline{\boldsymbol{s}} \otimes \overline{\boldsymbol{n}},
$$

in terms of the unit vector $\overline{\boldsymbol{n}}$ normal to the band, and the unit vector $\overline{\boldsymbol{s}}$ which defines the type of discontinuity across the matrix-band interface. For a general discontinuity, we have $0 \leqslant|\bar{s} \cdot \bar{n}| \leqslant 1$. Shear bands are a special case characterized by $\overline{\boldsymbol{s}} \cdot \overline{\boldsymbol{n}}=0$.

Every analysis using the present sub-grid method begins with material in the virgin state (i.e. without pre-existing localization bands). At that stage, the conventional and sub-grid formulations are equivalent and produce identical results. With the sub-grid method, however, the perturbation analysis of Sec. 2.6 is performed every time step, at each Gaussian quadrature point representing virgin material, to test for incipient shear localization. After the onset of localization behavior is detected at a given quadrature point, the local $\tilde{\mathbf{B}}$ operator is updated every time step using Eq. (61). This requires the three local quantities $\overline{\boldsymbol{n}}, \overline{\boldsymbol{s}}$, and $\alpha^{m}$ to be evaluated. The unit normal to the band is computed first, using the push-forward operation

$$
\overline{\boldsymbol{n}}=\frac{\mathbf{F}_{*}^{-\mathrm{T}} \overline{\boldsymbol{n}}_{*}}{\left\|\mathbf{F}_{*}^{-\mathrm{T}} \overline{\boldsymbol{n}}_{*}\right\|},
$$

which accounts for rotation of the band under large deformation. Here, $\overline{\boldsymbol{n}}_{*}$ is the unit normal vector representing the initial band orientation, obtained from the perturbation analysis, and

$$
\mathbf{F}_{*}:=\frac{\partial \boldsymbol{x}}{\partial \boldsymbol{X}_{*}}
$$


where $\boldsymbol{X}_{*}$ is the configuration where localization was initiated. Traction continuity (equilibrium) at the matrix-band interface requires

$$
\left(\boldsymbol{\sigma}^{b}-\boldsymbol{\sigma}^{m}\right) \cdot \overline{\boldsymbol{n}}=\mathbf{0},
$$

where $\sigma^{b}$ and $\sigma^{m}$ denote the local stress in the band and matrix materials, respectively. This nonlinear equation is solved for the remaining two quantities, $\alpha^{m}$ and $\bar{s}$, with the aid of a local Newton iteration.

\subsection{Time integration scheme}

Partitioning the interval $\mathcal{T}$ into increments $\left[t_{(n)}, t_{(n+1)}\right]$ of size $\Delta t$, we denote by $(\bullet)_{(n)}$ and $(\bullet)_{(n+1)}$ quantities evaluated at the beginning and at the end of the increment, respectively. The explicit central difference scheme is used to update the nodal solution variables, starting from their known values at $t=t_{(n)}$ :

$$
\begin{aligned}
& \mathbf{d}_{(n+1)}=\mathbf{d}_{(n)}+\dot{\mathbf{d}}_{(n)} \Delta t+\frac{1}{2} \ddot{\mathbf{d}}_{(n)} \Delta t^{2}, \\
& \dot{\mathbf{d}}_{(n+1)}=\dot{\mathbf{d}}_{(n)}+\frac{1}{2}\left(\ddot{\mathbf{d}}_{(n)}+\ddot{\mathbf{d}}_{(n+1)}\right) \Delta t,
\end{aligned}
$$

where the updated nodal accelerations are obtained from Eq. (51), after replacing the consistent mass matrix $\mathbf{M}$ by its lumped counterpart $\hat{\mathbf{M}}$ for improved computational efficiency, i.e.,

$$
\ddot{\mathbf{d}}_{(n+1)}=\hat{\mathbf{M}}^{-1}\left\{\mathbf{f}_{(n+1)}^{\mathrm{ext}}-\mathbf{f}_{(n+1)}^{\mathrm{int}}\right\} .
$$

It is noted that the external force vector $\mathbf{f}_{(n+1)}^{\text {ext }}$ is evaluated using Eq. (53) in a straightforward manner, since $\overline{\boldsymbol{h}}(\boldsymbol{x}, t)$ is a known function. On the other hand, in the conventional formulation, $\mathbf{f}_{(n+1)}^{\text {int }}$ is obtained using Eq. (55), i.e.

$$
\mathrm{f}_{B}^{\mathrm{int}}{ }_{(n+1)}=\sum_{e=1}^{n_{\mathrm{e}}} \int_{\Omega^{e}} \overline{\mathrm{B}}_{i j B}{ }_{(n+1)} \sigma_{i j}{ }_{(n+1)} \mathrm{d} \Omega,
$$

where $\overline{\mathbf{B}}_{(n+1)}$ involves gradients of the basis functions with respect to the configuration $\boldsymbol{x}_{(n+1)}$. Note that in the sub-grid formulation, $\overline{\mathbf{B}}_{(n+1)}$ is replaced by $\tilde{\mathbf{B}}_{(n+1)}$ in the above expression; see Eq. (60). In both formulations, the updated stress $\sigma_{(n+1)}$ is computed using the algorithms discussed in Sec. 3.4.

\subsection{Integration of the constitutive relations}

The constitutive update procedure adopted herein is very similar to the algorithm described in detail in a previous publication (Mourad et al., 2014). The key difference is that, in the present work, we use a mid-point rule formulated in the corotational description to integrate the constitutive relations (14); viz.

$$
\boldsymbol{\Sigma}_{(n+1)}=\boldsymbol{\Sigma}_{(n)}+\Delta t \dot{\boldsymbol{\Sigma}}_{\left(n+\frac{1}{2}\right)},
$$

instead of using the formula of Hughes and Winget (1980), which is formulated entirely in the spatial description. We employ the classical radial return algorithm, which consists of an elastic predictor,

$$
\boldsymbol{\Sigma}^{*}=\boldsymbol{\Sigma}_{(n)}+\mathbb{C}^{\mathrm{e}}:\left(\boldsymbol{\Lambda}_{\left(n+\frac{1}{2}\right)}^{\mathrm{T}} \cdot \boldsymbol{\Delta} \mathbf{d}_{\left(n+\frac{1}{2}\right)} \cdot \boldsymbol{\Lambda}_{\left(n+\frac{1}{2}\right)}\right),
$$

followed by a viscoplastic corrector,

$$
\boldsymbol{\Sigma}_{(n+1)}=\boldsymbol{\Sigma}^{*}-2 G \Delta \lambda \mathbf{N}_{(n+1)},
$$

which projects the elastic trial state back onto the yield surface, if needed, by solving the nonlinear equation (18) for the viscoplastic multiplier increment, $\Delta \lambda$. This is achieved using a Newton iterative scheme where the linearized equation

$$
\mathcal{F}^{(k+1)} \approx \mathcal{F}^{(k)}+\frac{d \mathcal{F}^{(k)}}{d \Delta \lambda^{(k)}}\left(\Delta \lambda^{(k+1)}-\Delta \lambda^{(k)}\right)=0,
$$


is solved in iteration $(k+1)$. Linearization of the MTS model equations is discussed in detail by Mourad et al. (2014). Finally, the spatial Cauchy stress is obtained via the inverse of the transformation (15):

$$
\sigma_{(n+1)}=\Lambda_{(n+1)} \cdot \boldsymbol{\Sigma}_{(n+1)} \cdot \Lambda_{(n+1)}^{\mathrm{T}} .
$$

In accordance with Eq. (11), the rotation tensors in the above relations are given by

$$
\begin{aligned}
\boldsymbol{\Lambda}_{\left(n+\frac{1}{2}\right)} & =\mathbf{Q} \Lambda_{(n)}, \\
\boldsymbol{\Lambda}_{(n+1)} & =\mathbf{Q} \Lambda_{\left(n+\frac{1}{2}\right)},
\end{aligned}
$$

where $\mathbf{Q}$ is obtained via exponential mapping (see Simo and Hughes, 1998, Sec. 8.3, for details):

$$
\mathbf{Q}=\exp \left[\Delta \mathbf{w}_{\left(n+\frac{1}{2}\right)}\right] .
$$

In addition, based on (2)-(3), we have

$$
\begin{aligned}
\Delta \mathbf{d}_{\left(n+\frac{1}{2}\right)} & =\frac{1}{2}\left(\Delta \mathbf{l}_{\left(n+\frac{1}{2}\right)}+\Delta \mathbf{l}_{\left(n+\frac{1}{2}\right)}^{\mathrm{T}}\right), \\
\Delta \mathbf{w}_{\left(n+\frac{1}{2}\right)} & =\frac{1}{2}\left(\Delta \mathbf{l}_{\left(n+\frac{1}{2}\right)}-\Delta \mathbf{l}_{\left(n+\frac{1}{2}\right)}^{\mathrm{T}}\right),
\end{aligned}
$$

where, in the conventional finite element formulation,

$$
\Delta \mathbf{l}_{\left(n+\frac{1}{2}\right)}:=\overline{\mathbf{B}}_{\left(n+\frac{1}{2}\right)}\left(\mathbf{d}_{(n+1)}-\mathbf{d}_{(n)}\right),
$$

and in the sub-grid formulation, $\overline{\mathbf{B}}_{\left(n+\frac{1}{2}\right)}$ is replaced by $\tilde{\mathbf{B}}_{\left(n+\frac{1}{2}\right)}$ in the above expression, cf. (59).

\section{Experimental procedures}

\subsection{Shear localization tests and top-hat specimens}

The cylindrical hat-shaped specimen developed by Meyers and Manwaring (1986) was used to conduct highdeformation forced-shear experiments in a variety of materials (Bronkhorst et al., 2006; Cerreta et al., 2009, 2013). The sample design and configuration used lead to a complex loading state, given the oblique orientation of the shear zone relative to the loading direction. It has been used for a number of years to effectively study the response of metallic materials to shear-dominated deformation, while suppressing the formation of cavities at the later stages of shear loading. In the current study, AISI 316L stainless steel samples were tested in this forced-shear configuration. A schematic drawing of the specimen is shown in Fig. 1 with specific sample dimensions given in Table 1. Meyers et al. (1995) described the details of the loading geometry. Controlled levels of deformation were imparted to specimens loaded dynamically in the split-Hopkinson pressure bar (SHPB) test system. One series of tests was performed on annealed samples using a number of striker bar lengths and breach pressures to vary deformation rate and pulse duration. These tests were performed at top surface displacement rates ranging from 8 to $25 \mathrm{~m} / \mathrm{s}$, test durations between 35 and $70 \mu \mathrm{s}$, and initial temperatures of $295 \mathrm{~K}$. One experiment belonging to this series is labeled Experiment $A$, and is conducted using a striker bar length of $15.24 \mathrm{~cm}$ and breech gas pressure of $174 \mathrm{kPa}$. Another suite of tests, labeled Experiment $B$, was performed on a series of identical samples with tool steel collars placed around the outside of the sample in order to avoid overdrive and to arrest the deformation at predetermined displacements. This setup facilitates the metallographic study of the progression of the adiabatic shear banding process. In experiment $\mathrm{B}$, dynamic testing was performed at an initial temperature of $298 \mathrm{~K}$, a breech gas pressure of $42 \mathrm{kPa}$ and a striker bar length of $15.24 \mathrm{~cm}$.

It is noted that the inherent oscillations in the dynamic stress-strain curves and lack of stress and strain equilibrium in the specimens at low strains make the determination of yield inaccurate at high strain rates. Since we are interested primarily in the late-time response of these samples, this does not in any way compromise the conclusions drawn from these experiments. 


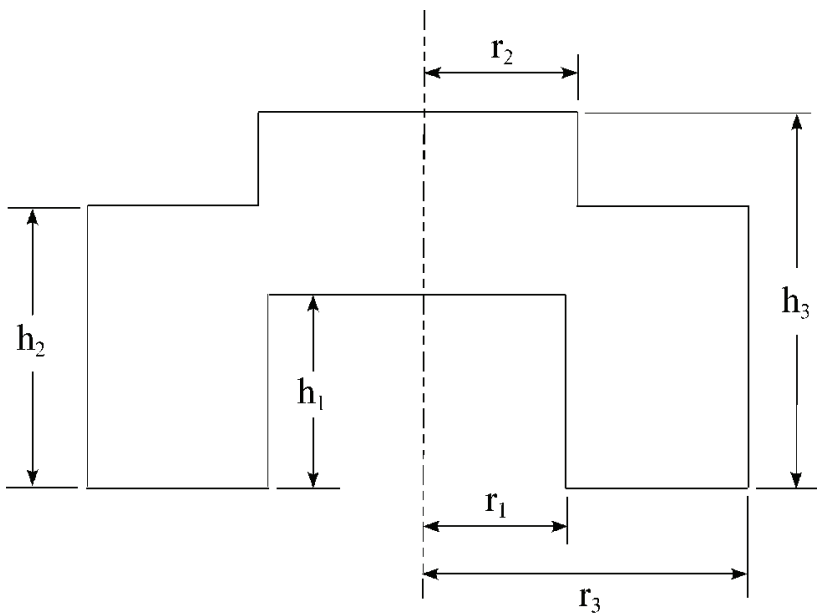

Figure 1: Schematic drawing of the axi-symmetric hat-shaped sample. Drawing is only approximately to scale.

Table 1: Sample dimensions in mm. The dimension variables correspond to those in Fig. 1.

\begin{tabular}{|c|c|c|c|c|c|}
\hline$r_{1}$ & $r_{2}$ & $r_{3}$ & $h_{1}$ & $h_{2}$ & $h_{3}$ \\
\hline 2.095 & 2.285 & 4.320 & 2.540 & 3.430 & 5.080 \\
\hline
\end{tabular}

Table 2: Composition of the 316L stainless steel (in wt. \%)

\begin{tabular}{|c|c|c|c|c|c|c|c|c|c|c|c|}
\hline $\mathbf{C}$ & $\mathbf{C r}$ & $\mathbf{C u}$ & $\mathbf{C o}$ & $\mathbf{M n}$ & $\mathbf{M o}$ & $\mathbf{N i}$ & $\mathbf{P}$ & $\mathbf{S}$ & $\mathbf{S i}$ & $\mathbf{N}$ & $\mathbf{F e}$ \\
\hline 0.022 & 16.16 & 0.39 & 0.19 & 1.70 & 2.08 & 10.03 & 0.029 & 0.0004 & 0.4 & 0.063 & Bal. \\
\hline
\end{tabular}




\subsection{AISI 316L stainless steel material}

The material was procured in $12.5 \mathrm{~mm}$-thick plate form that was hot-rolled and cooled in air and subsequently annealed. The composition of this material is described in Table 2 . The average grain size was approximately $30 \mu \mathrm{m}$. Although the grains were equi-axed and pole figures show a mild initial crystallographic texture, the inclusion and precipitate traces were parallel to the plane of the plate. The axi-symmetric specimens were machined from the plate with their axis parallel to the plate normal. The specimens were recovered after the tests, sectioned across the diameter, and prepared using classic metallographic techniques. The shear zone was imaged in each specimen using light optical microscopy.

\section{Results and discussion}

As was pointed out in previous sections, the ability to predict the formation and growth of adiabatic shear bands, and to represent this process in a computational setting, requires specialized methods capable of rendering the topology of the band region, and also requires the physics of the softening processes to be represented. Here, we propose that both the increased mobility of dislocations due to temperature increase and the recovery of history-dependent dislocation structure in this high rate loading condition play necessary roles in this process. In the following, we present simulation results corresponding to varying model parameters and computational conditions, and compare these to observations from experiments A and B, outlined in Sec. 4, to assess the suitability of the proposed theoretical and computational approach for this high-deformation rate problem.

The split-Hopkinson pressure bar (SHPB) experimental system allows for extraction of the change in the sample's overall height over the duration of the experiment. The velocity of the sample's top surface in each experiment is plotted against time in Fig. 2 (black curves). The oscillations in these velocity profiles are due to the interaction of stress waves traveling through the sample. The red curves in Fig. 2 are approximations of these velocity profiles, applied as boundary conditions in the numerical simulations to enable direct comparison to experimental results. The two finite element meshes shown in Fig. 3 are used in this study: the first is a coarse mesh with an element size of $h=90 \mu \mathrm{m}$ within the shear section, and the second is more refined with $h=40 \mu \mathrm{m}$. The material parameters used throughout the present work are listed in Table 3. These parameters, originally obtained from quasi-static and dynamic simple compression test data (not presented here), have been published and used in previous work by Bronkhorst et al. (2006).

\subsection{Experiment $A$}

First, we attempt to reproduce the results of experiment A using the classical MTS model, Eqs. (24)-(33), i.e. taking thermal softening into account, but not dynamic recrystallization. The conventional finite element formulation is used at two different levels of mesh refinement (Fig. 3). The resulting load-displacement curves exhibit very similar hardening behavior in clear disagreement with experimental data, as can be seen from Fig. 4a. This demonstrates that thermal softening, without additional softening mechanisms such as dynamic recrystallization, cannot account for the loss in load-carrying capacity observed experimentally. To explore this idea further, the computations are repeated using the DRX model of Sec. 2.5, in order to include the effect of dynamic recrystallization in addition to the thermal softening effect. The resulting load-displacement curves, produced using a refined mesh $(h=40 \mu \mathrm{m})$ of conventional elements, and a coarse mesh $(h=90 \mu \mathrm{m})$ of sub-grid elements with an embedded localization band of width $\omega=40 \mu \mathrm{m}$, are presented in Fig. 4b. Both curves display comparable softening response.

Another comparison between results obtained using the classical MTS model (considering thermal softening only) and those obtained using the DRX model (accounting for both thermal softening and dynamic recrystallization) is presented in Fig. 5. The sample's cross-sectional deformed shape and contour plots of logarithmic shear strain are shown to emphasize the extent of strain localization predicted with each model. All results shown in this figure are computed using a refined mesh $(h=40 \mu \mathrm{m})$ of conventional elements. Together with Fig. 4, comparisons of cross-sectional deformation with the metallographic image in Fig. 5e strongly suggest that both thermal softening and dynamic recrystallization are active mechanisms within this class of stainless steel materials under the dynamic loading conditions being considered. This is consistent with the growing evidence of the importance of dynamic recrystallization as a softening mechanism, as articulated in Sec. 1. 


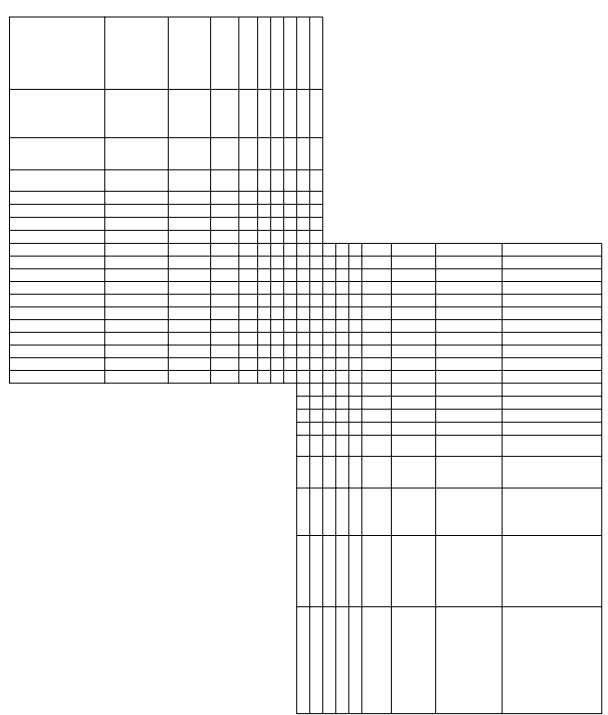

(a) Coarse mesh with $h=90 \mu \mathrm{m}$ in the shear section

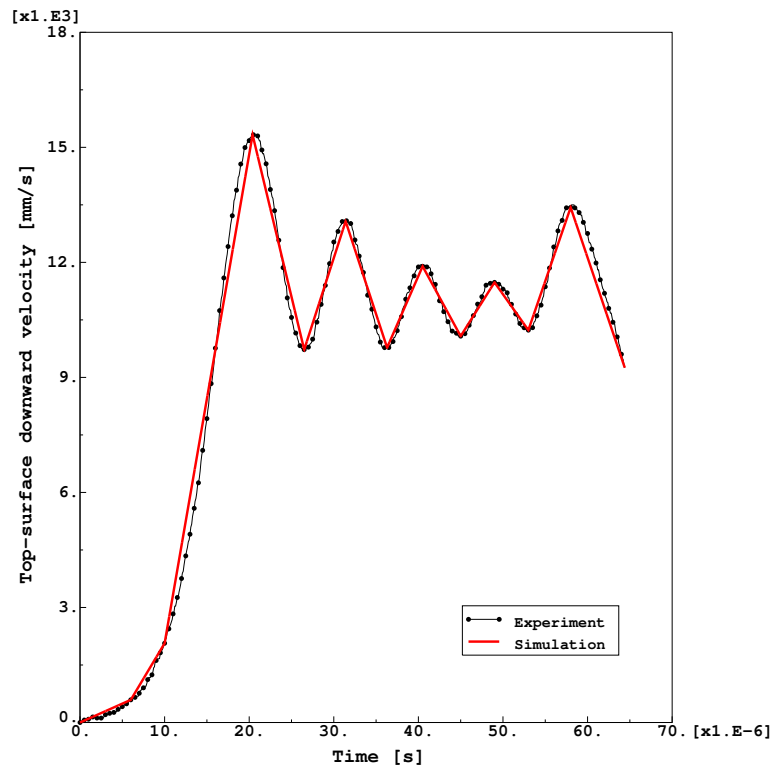

(b) Experiment B

Figure 2: Downward velocity profiles imposed on the top surface of the sample in experiments A and B. The experimentally observed velocity profiles are shown along with the profiles applied as boundary conditions in the computational simulations.

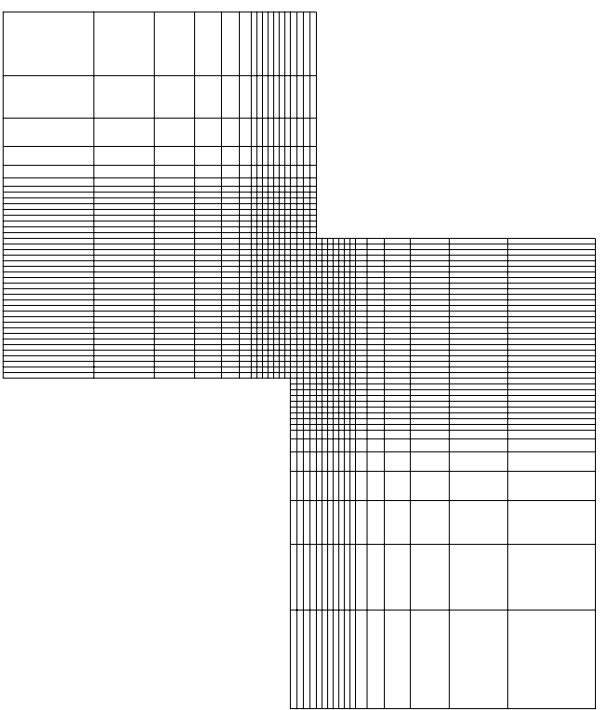

Figure 3: Meshes of axi-symmetric finite elements used in the current study. 
Table 3: Material parameters for 316L stainless steel (Bronkhorst et al., 2006)

\begin{tabular}{|c|c|c|}
\hline Parameter & Value & Unit \\
\hline$\rho$ & 7860 & $\mathrm{~kg} / \mathrm{m}^{3}$ \\
\hline$K$ & 158 & $\mathrm{GPa}$ \\
\hline$G$ & 73 & $\mathrm{GPa}$ \\
\hline$\theta_{\text {melt }}$ & 1670 & $\mathrm{~K}$ \\
\hline$\hat{\sigma}_{a}$ & 40 & $\mathrm{MPa}$ \\
\hline$\mu_{0}$ & 71.46 & $\mathrm{GPa}$ \\
\hline$D_{0}$ & 2.91 & $\mathrm{GPa}$ \\
\hline$\theta_{0}$ & 204 & $\mathrm{~K}$ \\
\hline$b$ & $2.48 \times 10^{-10}$ & $\mathrm{~m}$ \\
\hline$\dot{\varepsilon}_{0 i}, \dot{\varepsilon}_{0 \epsilon}, \dot{\varepsilon}_{0 \epsilon s}$ & $10^{7}$ & $\mathrm{~s}^{-1}$ \\
\hline$q_{i}$ & $3 / 2$ & - \\
\hline$p_{i}$ & $1 / 2$ & - \\
\hline$g_{0 i}$ & 0.325 & - \\
\hline$\hat{\sigma}_{i}$ & 450 & $\mathrm{MPa}$ \\
\hline$q_{\epsilon}$ & 1 & - \\
\hline$p_{\epsilon}$ & $2 / 3$ & - \\
\hline$g_{0 \epsilon}$ & 1.6 & - \\
\hline$\hat{\sigma}_{\epsilon}$ (initial value) & 255 & $\mathrm{MPa}$ \\
\hline$\kappa$ & 1.8 & - \\
\hline$A_{0}$ & 4.1 & $\mathrm{GPa}$ \\
\hline$A_{3}$ & 3.25 & $\mathrm{MPa} / \mathrm{K}$ \\
\hline$A_{1}, A_{2}, A_{4}, A_{5}$ & 0 & - \\
\hline$g_{0 \epsilon s}$ & 0.165 & - \\
\hline$\hat{\sigma}_{\epsilon s 0}$ & 3.475 & $\mathrm{GPa}$ \\
\hline$\Psi$ & 0.95 & - \\
\hline$C_{0}$ & 391.63 & $\mathrm{~J} / \mathrm{kg} \mathrm{K}$ \\
\hline$C_{1}$ & 0.237 & $\mathrm{~J} / \mathrm{kg} \mathrm{K}^{2}$ \\
\hline$C_{2}$ & 0 & $\mathrm{~J} \mathrm{~K} / \mathrm{kg}$ \\
\hline$D_{\mathrm{drx}}$ & 0.04 & $\mathrm{~mm}^{2} / \mathrm{s}$ \\
\hline$Q$ & 22 & $\mathrm{~kJ} / \mathrm{mol} \mathrm{K}$ \\
\hline$\tilde{d}$ & 5 & $\mu \mathrm{m}$ \\
\hline$\theta_{\mathrm{drx}}$ & 355 & $\mathrm{~K}$ \\
\hline
\end{tabular}

Figures 6 and 7 present contours of accumulated plastic strain and temperature, respectively, obtained using the sub-grid formulation coupled with the DRX model. Here, Figs. $6 \mathrm{~b}$ and $7 \mathrm{~b}$ show the state of the material inside the embedded localization band, with elements shown in gray representing virgin material (i.e. free of shear bands), while Figs. $6 \mathrm{a}$ and $7 \mathrm{a}$ show the state of the material outside the band. As expected, more plastic deformation and higher temperatures are achieved within the shear band. Recall that conventional computational formulations yield meshdependent results in localization problems, even when used with a rate sensitive material, unless localization bands are explicitly resolved via mesh refinement. However, the computational cost associated with the required level of mesh refinement can be exorbitant in many cases. In addition, refined meshes are more prone to excessive distortion, especially in localization problems, requiring frequent remeshing and state variable field remapping. The sub-grid formulation is used here to mitigate these issues by embedding the localization band within the elements of the mesh. It also allows the use of a coarse mesh, in order to bring the numerical representation of shear band formation within practical reach. 


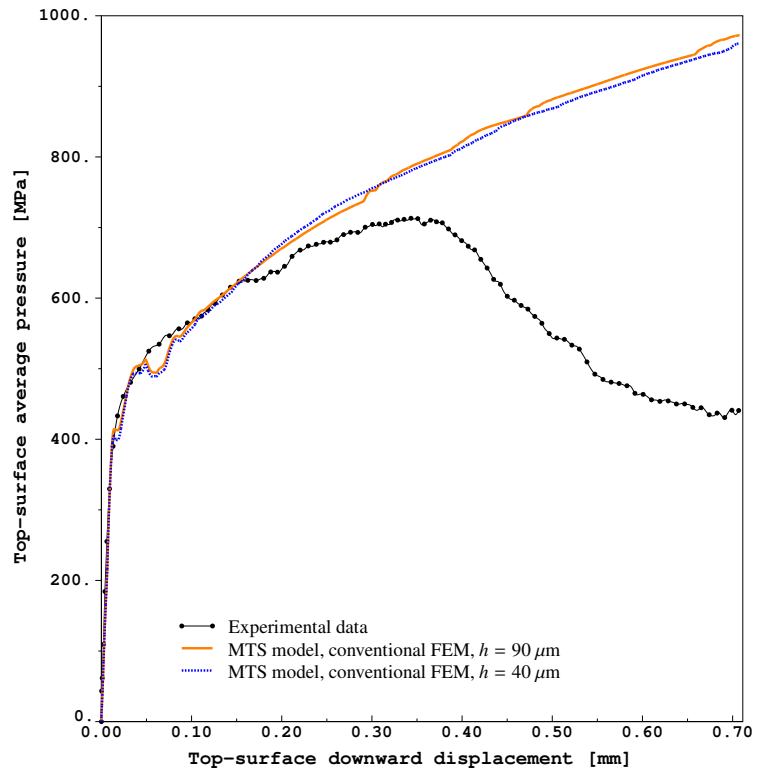

(a) MTS model results

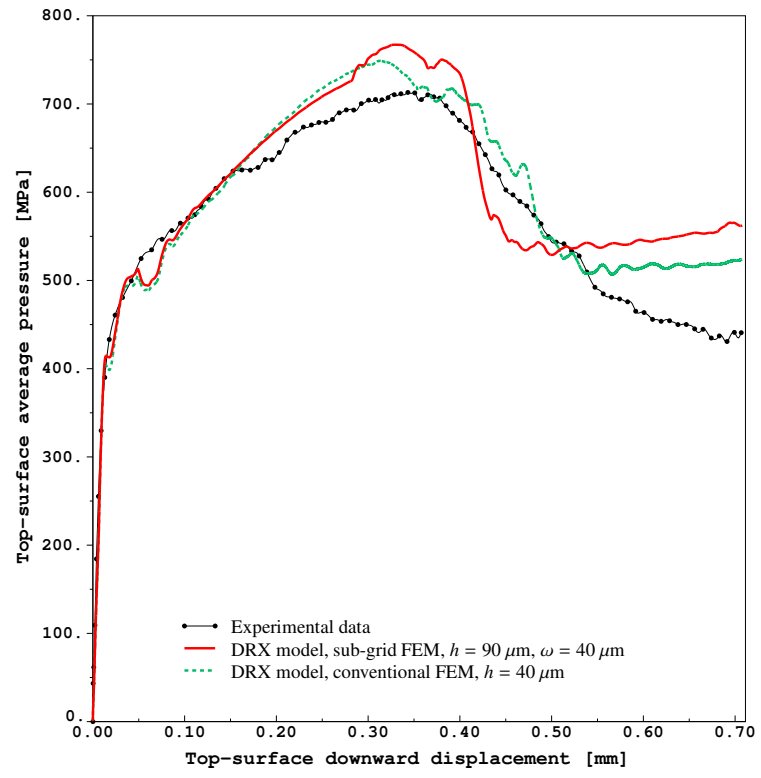

(b) DRX model results

Figure 4: Load-displacement curves computed using the classical MTS model (a) and the proposed DRX model (b), compared to measured data from experiment $\mathrm{A}$.

\subsection{Experiment $B$}

Turning our attention to experiment $\mathrm{B}$, we present a quantitative comparison between computational and experimental results in Fig. 8. The load-displacement response curves computed using the conventional finite element formulation, coupled with either the MTS or the DRX model, are compared in Fig. 8a. As expected, DRX model results shown in this figure exhibit mesh sensitivity due to softening, whereas the MTS model results appear almost independent of mesh size (i.e. converged). On the other hand, Fig. 8b presents computational results produced by the DRX model, in combination with the sub-grid formulation. The load-displacement curves shown in this figure correspond to three different values of the embedded shear band width, $\omega$, all falling within a physically meaningful range, consistent with experimental observations (see e.g. Xue and Gray III, 2006). This illustrates the dependence of the predicted response on this physical length scale. Furthermore, the results show that with reduced band thickness, softening is more rapid. This is consistent with expectation since localization of plastic work within a narrower band is expected to result in a higher local temperature within the band, thereby accelerating thermal softening and the (thermally activated) recrystallization process.

The deformed shape of the axi-symmetric sample is rendered in three dimensions in Fig. 9, with superposed contours of Mises stress. It is clear from this figure that the MTS and DRX models yield qualitatively different results. Importantly, shear band initiation in a fashion consistent with experimental observations is predicted only by the DRX model. The results in Fig. 9b also show a significant drop in the plastic flow stress in the shear band region, which explains the softening reflected in the load-displacement results.

Cross-sectional micrograph images at top surface displacements of $0.2,0.4$, and $0.6 \mathrm{~mm}$ are given in Fig. 10. These three images clearly show the gradual progression from homogeneous deformation to the initiation and propagation of a clear and distinctive shear band. For comparison, numerical results representing this progression are presented in Figs. 11a-e, showing snapshots of material state at top surface displacements of $0.231,0.272,0.331,0.354$, and 0.600 $\mathrm{mm}$, respectively. Contour plots of the structure dependent threshold stress, $\hat{\sigma}_{\epsilon}$, in the material outside the embedded band are shown (left) alongside contours of the same quantity within the embedded band material (right). Points representing these snapshots on the load-displacement history are shown in Fig. 11f. 


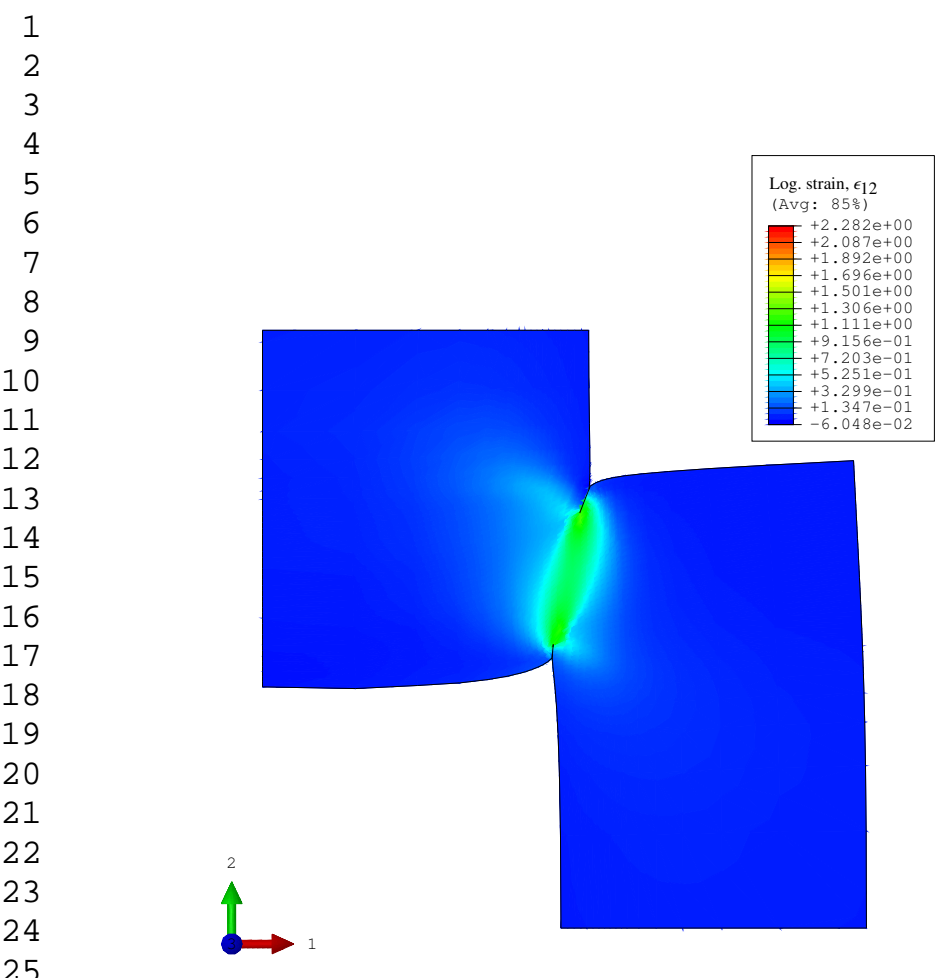

(a) MTS model results

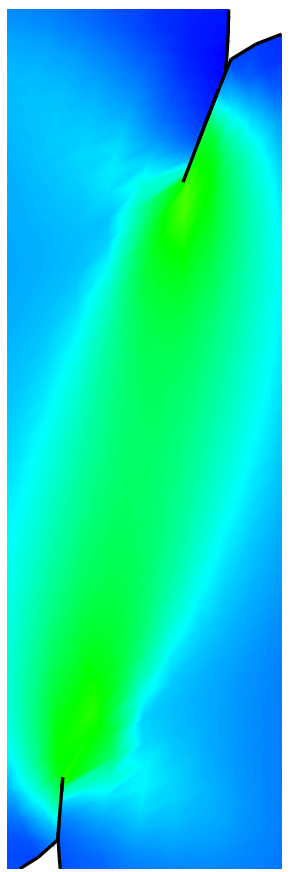

(c) Shear section (MTS)

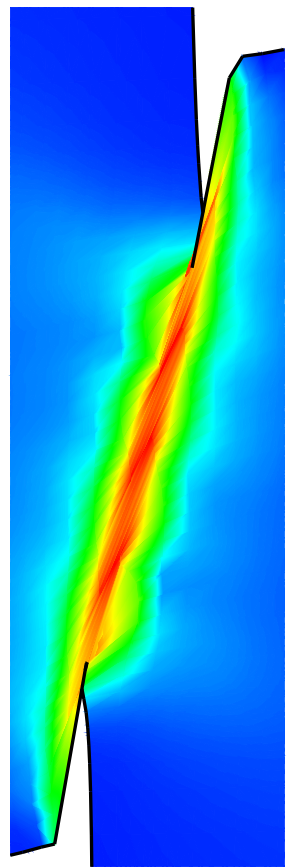

(d) Shear section (DRX)

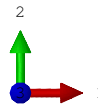

(b) DRX model results
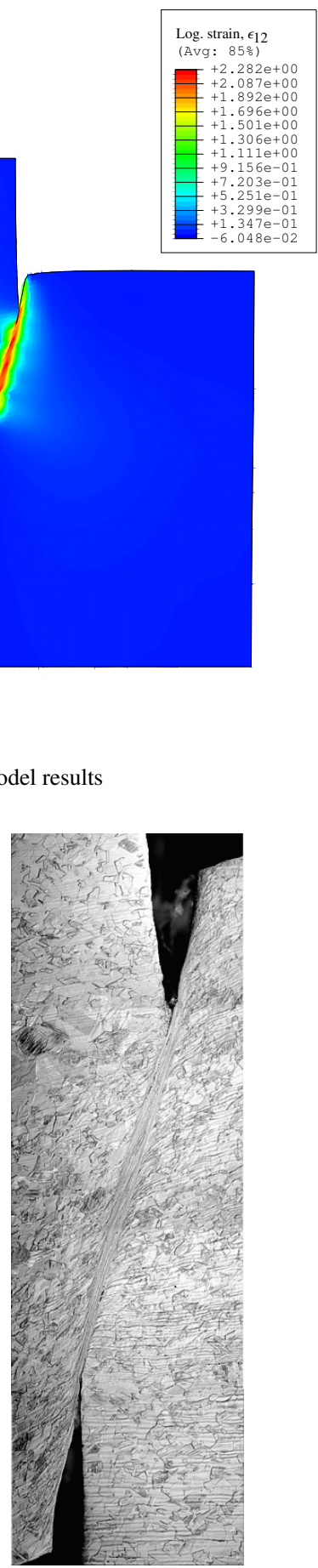

(e) Shear section micrograph

(Bronkhorst et al., 2006)

Figure 5: Logarithmic shear strain distribution at the end of experiment A, computed using a refined mesh of conventional elements $(h=40 \mu \mathrm{m}$, Fig. 3b). A closeup view of the shear section reveals that the DRX model produces significantly improved results (d), compared to MTS model results (c), in terms of qualitative agreement with previously published experimental observations (e). 


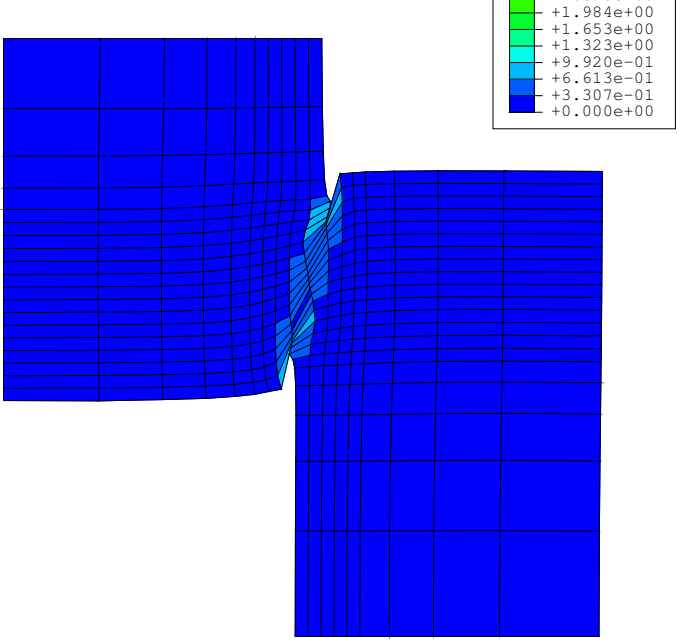

(a) Virgin/matrix material

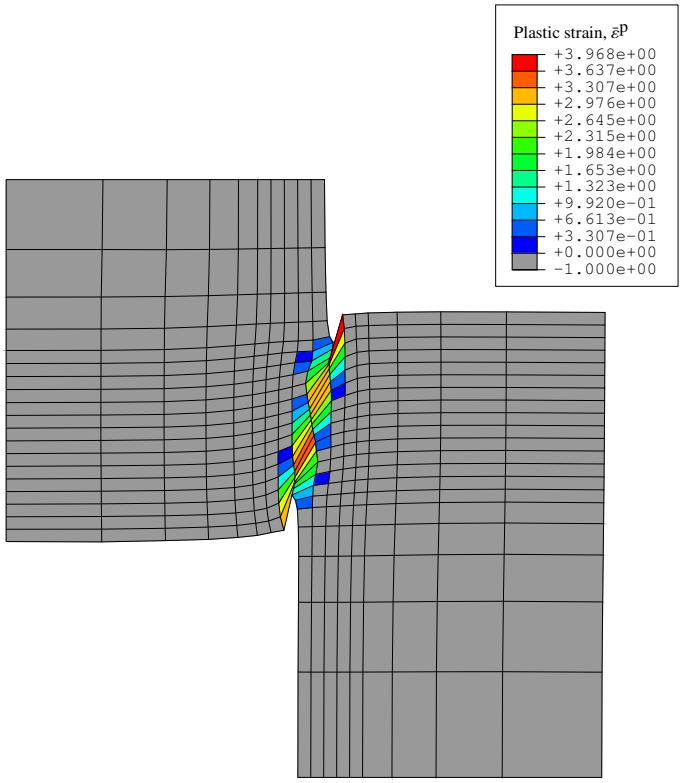

(b) Embedded shear band

Figure 6: Distribution of accumulated plastic strain at the end of experiment A, in the virgin/matrix material (a) and in the embedded shear band (b). The results shown were obtained using the DRX model and the sub-grid finite element method with $h=90 \mu \mathrm{m}, \omega=40 \mu \mathrm{m}$.

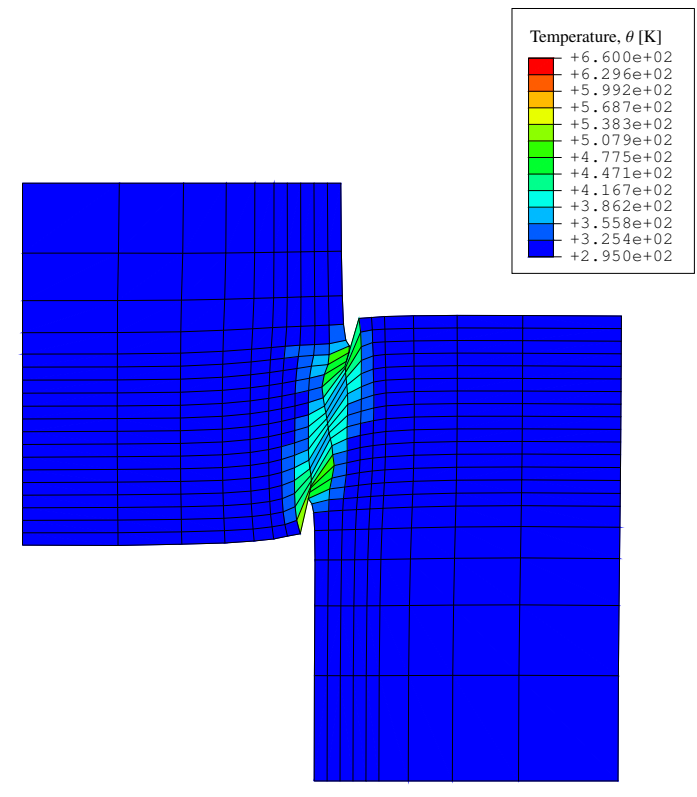

(a) Virgin/matrix material

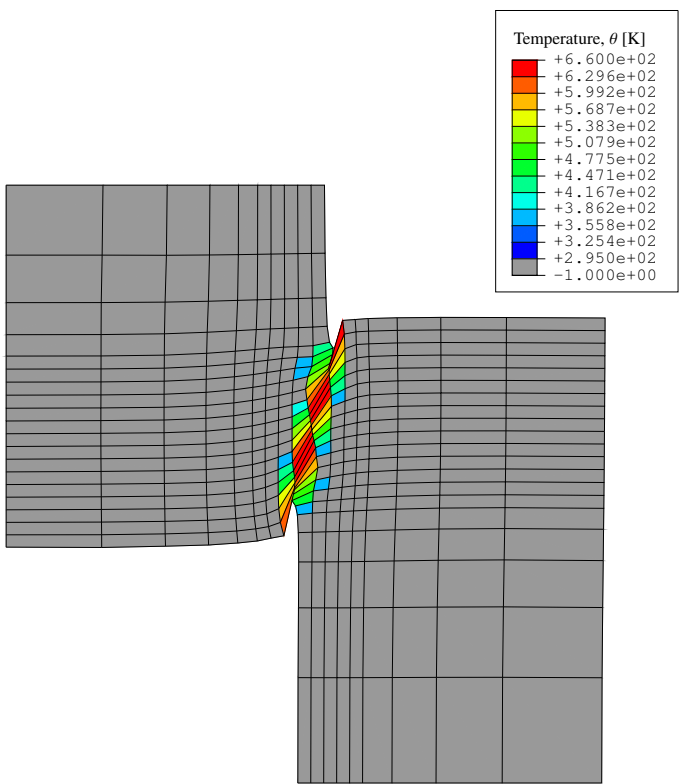

(b) Embedded shear band

Figure 7: Temperature distribution at the end of experiment A, in the virgin/matrix material (a) and in the embedded shear band (b). The results shown were obtained using the DRX model and the sub-grid finite element method with $h=90 \mu \mathrm{m}, \omega=40 \mu \mathrm{m}$. 


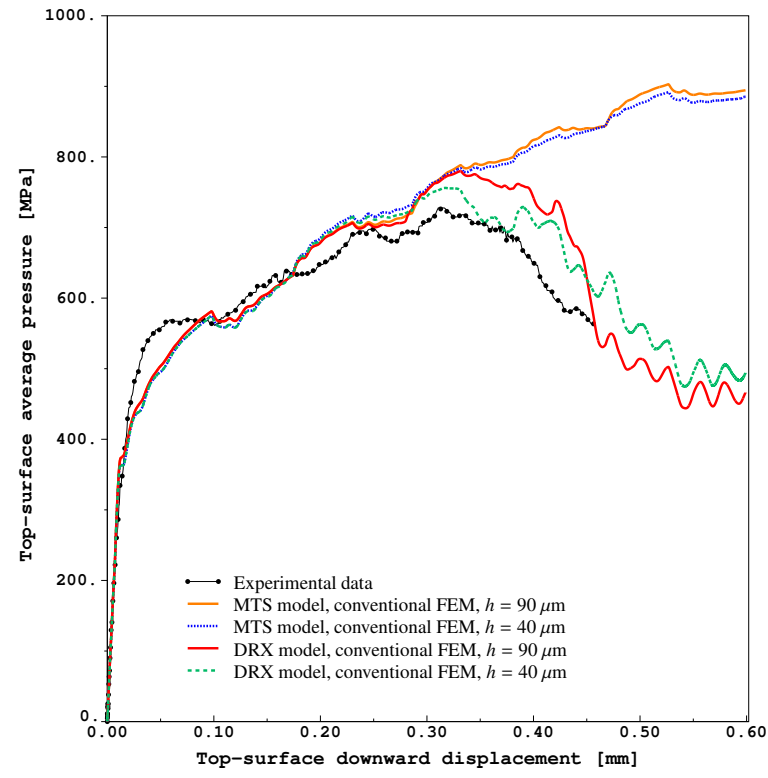

(a) Conventional FEM results

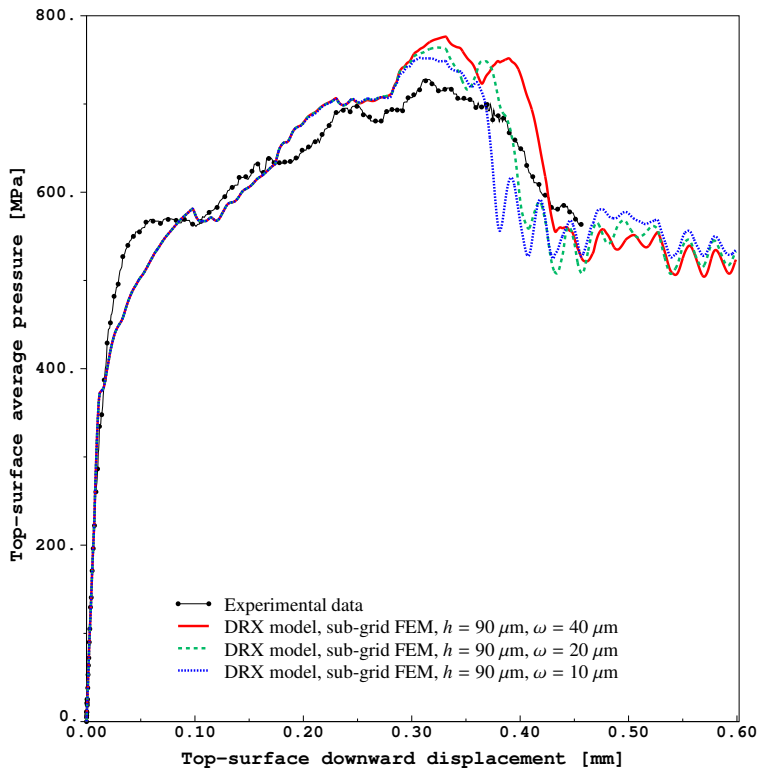

(b) Sub-grid FEM results

Figure 8: Load-displacement curves computed using conventional FEM (a) and sub-grid FEM (b), compared to measured data from experiment B.

\subsection{Discussion and outlook}

For many years, increased dislocation mobility due to the rise in temperature caused by mechanical work was thought to be the only softening mechanism responsible for adiabatic shear band nucleation and growth. The work presented here points to the importance of dynamic recrystallization as perhaps the dominant softening mechanism in a class of metallic materials, such as the AISI 316L stainless steel considered here. Although this paints a more complicated physical picture into this damage and failure process, it also offers new insights and a new opportunity to develop predictive tools for both commercial and defense applications.

The representation of dynamic recrystallization described here is a very simple one. However, it is believed to capture the essential rate effects, consistent with our understanding of dislocation motion and grain refinement under highly dynamic conditions (strain rates approaching $10^{5} \mathrm{~s}^{-1}$ are typical for the loading conditions considered here, as shown by Bronkhorst et al., 2006, 2007). It is important to keep in mind that our current understanding of the growth of new grains as a function of evolving dislocation structure and changing temperature, under these strongly driven conditions, is still very much incomplete. It is not yet possible to experimentally characterize the structural evolution occurring at sub-granular levels over the very short time scales associated with these processes. This will require more advanced characterization techniques. More meso-scale theory development will also be required to better articulate events and processes that take place within single crystals under these rapid loading conditions.

With dynamic recrystallization identified as a critical softening mechanism, materials can now be screened for their propensity to suffer damage and eventual failure due to this mechanism. New materials could potentially be designed to resist this type of recrystallization response, in order to delay failure and achieve greater levels of structural integrity.

The sub-grid finite element formulation presented here represents an extension of the embedded-zone elements of Belytschko et al. (1988) and Fish and Belytschko (1988) to the the case of dynamic problems involving rate-sensitive materials, where traditional material stability analysis methods, based on the eigenvalue decomposition of the acoustic tensor (Hill, 1962), are not applicable. The formulation is implemented within an explicit dynamic analysis framework and applied to a suite of highly dynamic problems. By embedding shear band segments within individual elements, this sub-grid technique produces significantly enhanced results in comparison to conventional finite element methods. However, there is ample room for improvement on several fronts. For example, ensuring the continuity of the embedded band along its entire length is expected to further improve the mesh-independent character of the results produced. Implementing this topological constraint is a non-trivial task that necessitates non-local (inter-element) information 


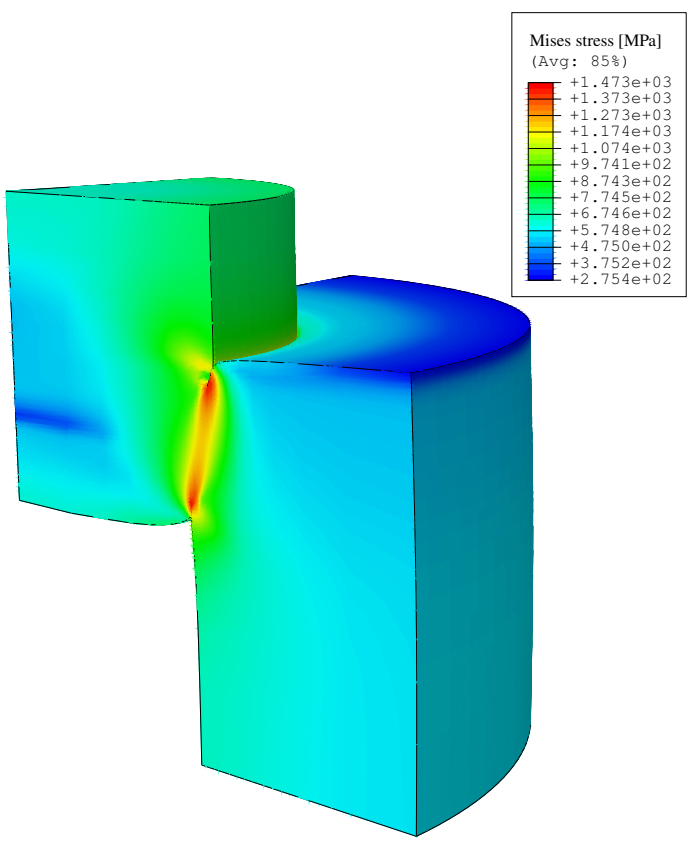

(a) MTS model results

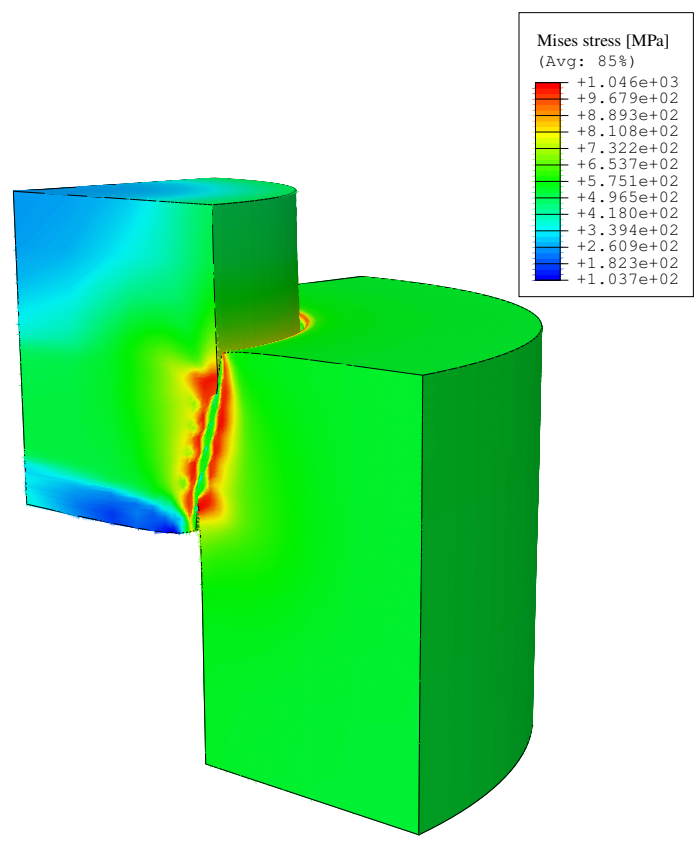

(b) DRX model results

Figure 9: Mises stress distribution at the end of experiment B, computed using a refined mesh of conventional elements $(h=40 \mu \mathrm{m})$, illustrating material softening due to dynamic recrystallization in the shear zone.

sharing. This is beyond the scope of the current work, and will be the focus of a future publication.

The experimental results of Fig. 10 illustrate the gradual evolution of the shear band width with deformation, and the numerical results of Fig. 8b demonstrate the potential importance of this effect which was not accounted for in the present study. Inclusion of this element of physics in the proposed framework will be carried out in future work. This will require a better understanding of how to represent the dimensional evolution of the shear band as a function of time and other variables, especially temperature. This will, in turn, necessitate multi-scale experimental and computational efforts, commensurate with the development of macro-scale and meso-scale material response theories, although the work of Plohr and Plohr (2016) can offer insights and perhaps a point of departure on this front.

In its present form, the proposed framework is limited to axial symmetry and material isotropy. A general threedimensional implementation will be pursued in the future, in order to gain the ability to predict the spatial location and timing of shear band initiation more accurately. The initiation of such damage and failure processes is strongly linked with the local defect variability within the material, as shown by Bronkhorst et al. (2016) in the context of void formation. Therefore, mean field representation of the material behavior will be inadequate in the future. The ability to represent the local-scale defect structure and its role in the shear band nucleation process will be a key community challenge.

\section{Summary and concluding remarks}

This paper presents a comprehensive framework for the treatment of adiabatic shear band formation and evolution within a computational setting. Gaining the ability to predict these complex damage and failure processes in macroscale problems is the ultimate goal of this work. Results obtained using the proposed modeling and simulation framework are compared against two forced-shear experiments performed on annealed 316L stainless steel samples loaded dynamically and are found to be very encouraging, suggesting that the proposed approach offers a sound and practical path toward achieving this challenging goal. Given the complexity of adiabatic shear band initiation and growth from physical and numerical perspectives, the framework introduced herein is a significant improvement over 


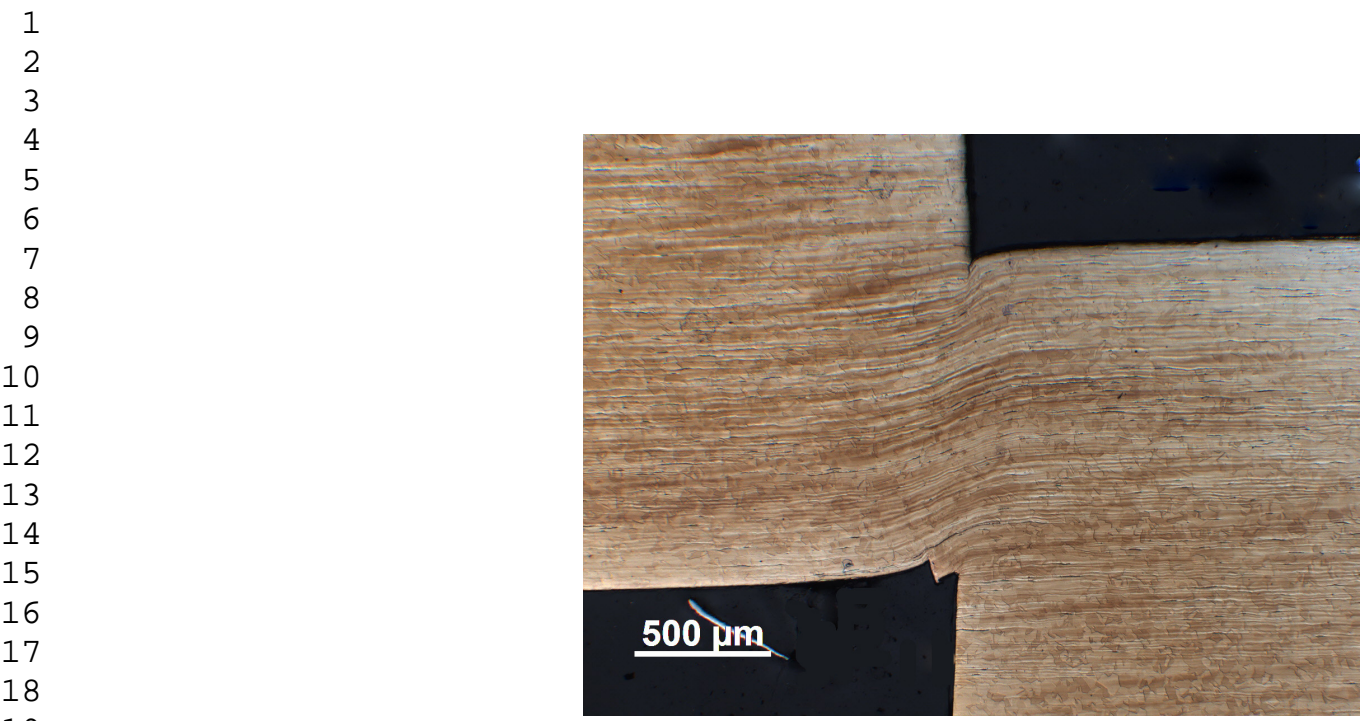

(a) Top-surface displacement: $0.2 \mathrm{~mm}$.

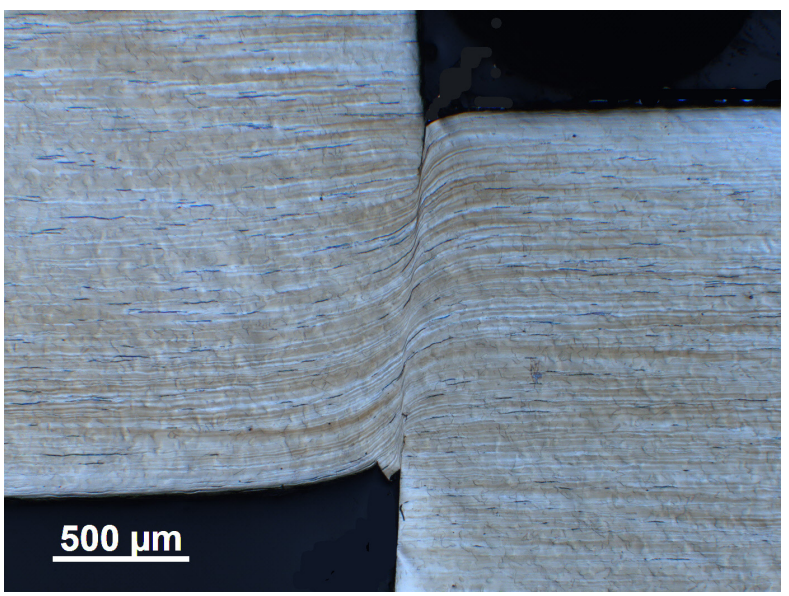

(b) Top-surface displacement: $0.4 \mathrm{~mm}$.

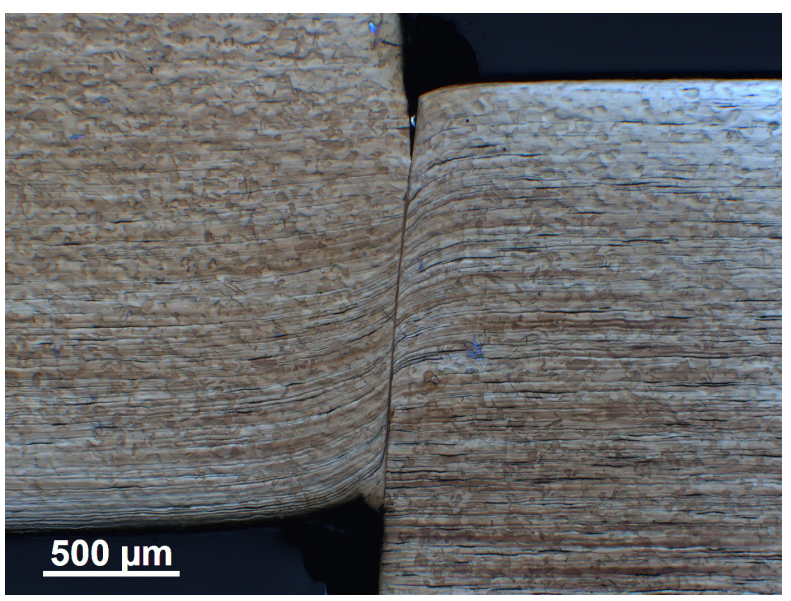

(c) Top-surface displacement: $0.6 \mathrm{~mm}$.

Figure 10: Micrographs showing the shear section at different stages of deformation in experiment B. 

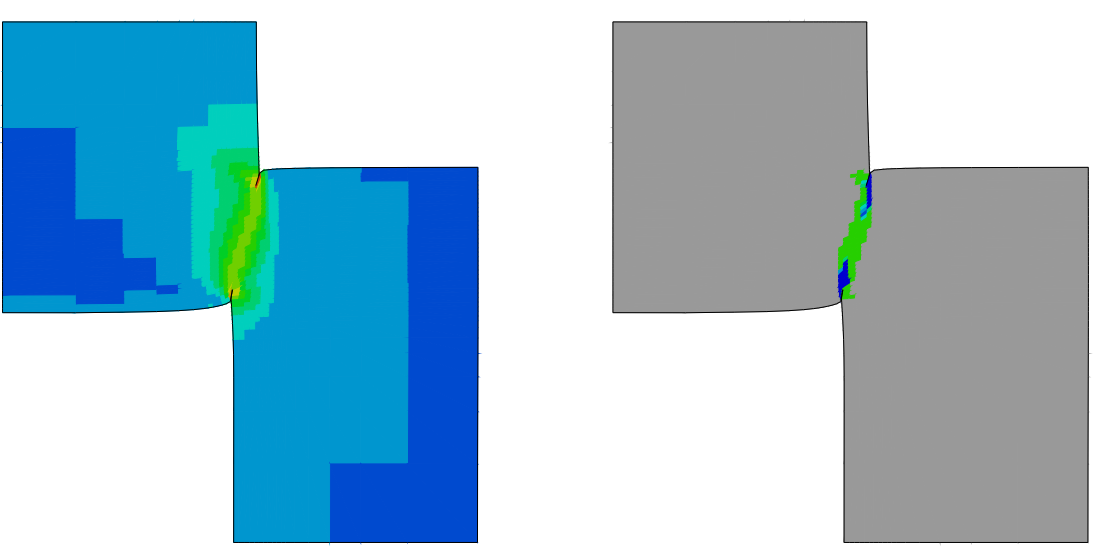

(c) Top-surface displacement: $0.331 \mathrm{~mm}$.

Figure 11: Contours of the structure-dependent threshold stress, $\hat{\sigma}_{\epsilon}$, in the virgin/matrix material (left) and the embedded band material (right), at five stages of deformation (a)-(e), shown on the corresponding load-displacement curve (f), to illustrate the concurrent progression of shear localization and dynamic recrystallization in experiment $\mathrm{B}$. These results were obtained using the the sub-grid formulation with $h=40 \mu \mathrm{m}$, $\omega=10 \mu \mathrm{m}$ (continued on next page). 

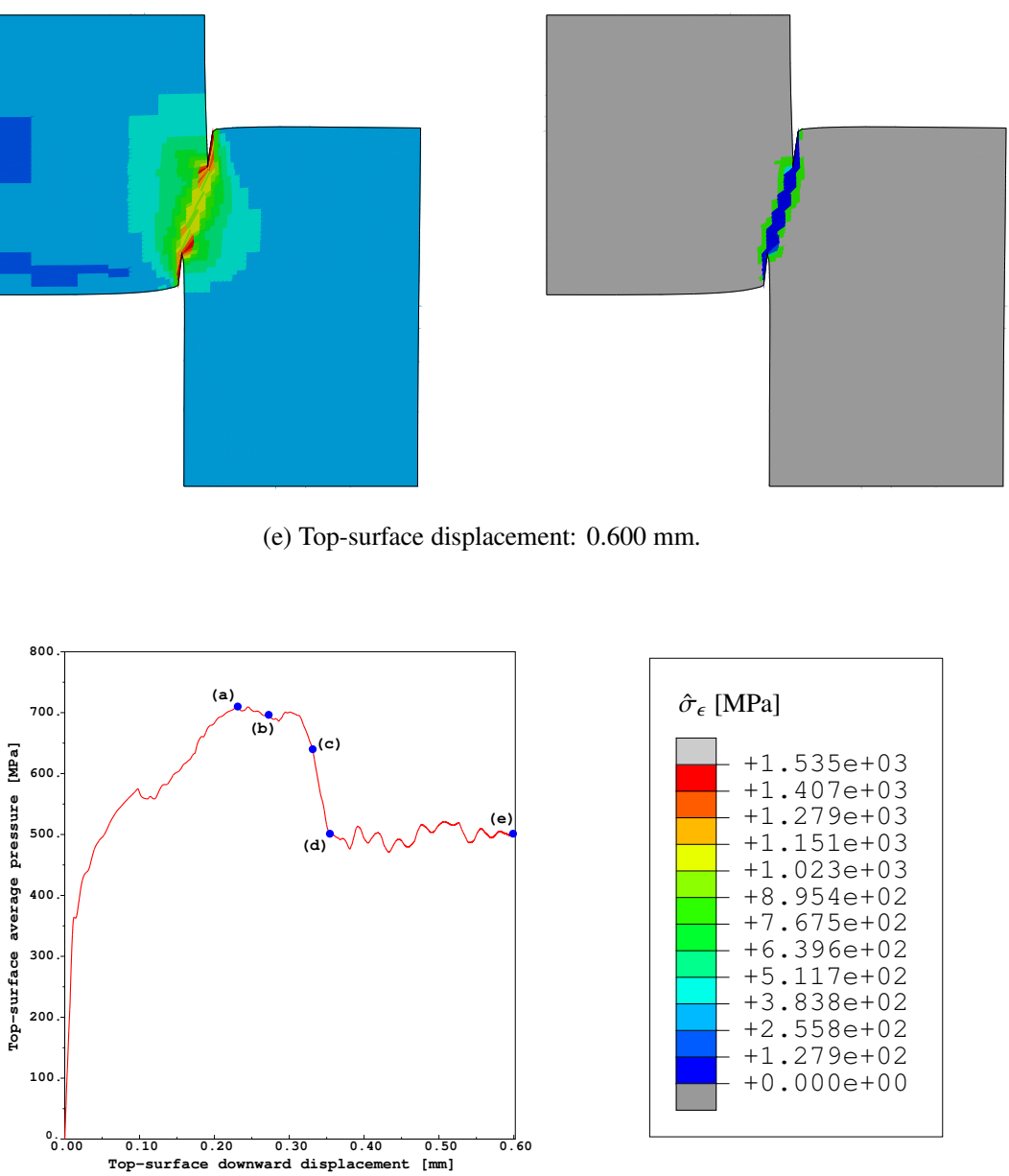

(e) Top-surface displacement: $0.600 \mathrm{~mm}$.

(f) Load-displacement curve and color legend

Figure 11: Contours of the structure-dependent threshold stress, $\hat{\sigma}_{\epsilon}$, in the virgin/matrix material (left) and the embedded band material (right), at five stages of deformation (a)-(e), shown on the corresponding load-displacement curve (f), to illustrate the concurrent progression of shear localization and dynamic recrystallization in experiment B. These results were obtained using the the sub-grid formulation with $h=40 \mu \mathrm{m}$, $\omega=10 \mu \mathrm{m}$. 
traditional ways of representing these rapid physical phenomena. However, despite the attention that this topic has received over many years, there is substantial, important work yet to be done in this area.

\section{Acknowledgments}

The authors wish to thank Dr. F. L. Addessio and Dr. J. F. Bingert for helpful discussions over the course of this work. We also wish to thank E. K. Walker for help with sample preparation. This work was funded by the DoD/DOE Joint Munitions Technology Development Program (Dr. T. A. Mason program manager), the NNSA Advanced Simulation and Computing-Physics and Engineering Models (ASC-PEM) program (Dr. D. L. Preston project leader, Dr. M. W. Schraad program manager), the NNSA Science Campaign 2-Dynamic Materials Properties (Dr. D. M. Dattelbaum program manager), and the Laboratory Directed Research and Development (LDRD) program at Los Alamos National Laboratory. This support is gratefully acknowledged.

Appendix A. Mitigation of mesh locking phenomena and stabilization of spurious zero-energy modes

Recalling that the $\mathbf{B}$ operator involves gradients of the shape functions, and is used to compute the velocity gradient tensor from the nodal velocities, i.e.

$$
\boldsymbol{\nabla} \dot{\boldsymbol{u}}^{h}=\mathbf{B} \dot{\mathbf{d}},
$$

we split B into symmetric and skew-symmetric components, which can be used to compute the rate of deformation tensor and the spin tensor, respectively:

$$
\mathbf{B}=\mathbf{B}^{\mathrm{sym}}+\mathbf{B}^{\mathrm{skw}} .
$$

Then, the symmetric part is split into volumetric and deviatoric parts,

$$
\mathbf{B}^{\mathrm{sym}}=\mathbf{B}^{\mathrm{vol}}+\mathbf{B}^{\mathrm{dev}},
$$

and the deviatoric part is split further into shear (off-diagonal) and normal (diagonal) parts:

$$
\mathbf{B}^{\mathrm{dev}}=\mathbf{B}^{\mathrm{shr}}+\mathbf{B}^{\mathrm{nrm}} .
$$

Combining Eqs. (A.2)-(A.4), we obtain

$$
\mathbf{B}=\mathbf{B}^{\mathrm{vol}}+\mathbf{B}^{\mathrm{shr}}+\mathbf{B}^{\mathrm{nrm}}+\mathbf{B}^{\mathrm{skw}} .
$$

The selective reduced integration technique of Hughes (1980) is then used to alleviate volumetric locking due to (nearly) incompressible material response. Selective reduced integration is also used to mitigate shear locking in bending dominated problems (e.g. see Belytschko and Bachrach, 1986). This is achieved by defining the $\overline{\mathbf{B}}$ operator,

$$
\overline{\mathbf{B}}=\mathbf{B}_{0}^{\mathrm{vol}}+\mathbf{B}_{0}^{\mathrm{shr}}+\mathbf{B}^{\mathrm{nrm}}+\mathbf{B}^{\mathrm{skw}}
$$

where, $\mathbf{B}_{0}^{\mathrm{vol}}$ and $\mathbf{B}_{0}^{\mathrm{shr}}$ are evaluated at the centroid of the four-noded quadrilateral elements used here, and treated as constant over the element. This is equivalent to the use of single-point quadrature for integration of volumetric and shear effects. Finally, to suppress hourglass modes that may pollute the solution as a result of using reduced quadrature rules, we redefine the $\overline{\mathbf{B}}$ operator as follows (see Ortiz et al., 1987):

$$
\overline{\mathbf{B}}=\left(1-\epsilon_{\mathrm{stb}}\right)\left[\mathbf{B}_{0}^{\mathrm{vol}}+\mathbf{B}_{0}^{\mathrm{shr}}\right]+\epsilon_{\mathrm{stb}}\left[\mathbf{B}^{\mathrm{vol}}+\mathbf{B}^{\mathrm{shr}}\right]+\mathbf{B}^{\mathrm{nrm}}+\mathbf{B}^{\mathrm{skw}},
$$

where $\epsilon_{\mathrm{stb}}$ is a small stabilization parameter. The value $\epsilon_{\mathrm{stb}}=0.05$ has been used throughout the present work. Note that setting $\epsilon_{\mathrm{stb}}=1$ recovers the conventional displacement-based formulation (A.5), whereas setting $\epsilon_{\mathrm{stb}}=0$ recovers the selective reduced integration technique (A.6). 


\section{References}

ABAQUS, 2014. Abaqus 6.14 Theory Guide. Dassault Systèmes Simulia Corp., Providence, RI, USA.

Abed, F., Makarem, F., 2012. Comparisons of constitutive models for steel over a wide range of temperatures and strain rates. J. Eng. Mater. Technol. 134, 021001-1-021001-10.

Abed, F. H., Makarem, F., Voyiadjis, G. Z., 2013. Dynamic localizations in HSLA-65 and DH-36 structural steel at elevated temperatures. J. Eng. Mater. Technol. 135, 021007-1 - 021007-11.

Abed, F. H., Voyiadjis, G. Z., 2005. Plastic deformation modeling of AL-6XN strainless steel at low and high strain rates and temperatures using a combination of bcc and fcc mechanisms of metals. Int. J. Plast. 21, 1618-1639.

Abu Al-Rub, R. K., Voyiadjis, G. Z., 2006. A physically based gradient plasticity theory. Int. J. Plast. 22, 654-684.

Anand, L., Aslan, O., Chester, S. A., 2012. A large-deformation gradient theory for elastic-plastic materials: strain softening and regularization of shear bands. Int. J. Plast. 30, 116-143.

Anand, L., Kim, K. H., Shawki, T. G., 1987. Onset of shear localization in viscoplastic solids. J. Mech. Phys. Solids 35, 407-429.

Armero, F., Kim, J., 2012. Three-dimensional finite elements with embedded strong discontinuities to model material failure in the infinitesimal range. Int. J. Numer. Methods Eng. 91, 1291-1330.

Armero, F., Linder, C., 2008. New finite elements with embedded strong discontinuities in the finite deformation range. Comput. Methods Appl. Mech. Eng. 197, 3138-3170.

Armero, F., Linder, C., 2009. Numerical simulation of dynamic fracture using finite elements with embedded discontinuities. Int. J. Fract. 160, $119-141$.

Arriaga, M., McAuliffe, C., Waisman, H., 2015. Onset of shear band localization by a local generalized eigenvalue analysis. Comput. Methods Appl. Mech. Eng. 289, 179-208.

Arriaga, M., McAuliffe, C., Waisman, H., 2016. Instability analysis of shear bands using the instantaneous growth-rate method. Int. J. Impact Eng. $87,156-168$.

Belytschko, T., Bachrach, W. E., 1986. Efficient implementation of quadrilaterals with high coarse-mesh accuracy. Comput. Methods Appl. Mech. Eng. 54, 279-301.

Belytschko, T., Fish, J., Engelmann, B. E., 1988. A finite element with embedded localization zones. Comput. Methods Appl. Mech. Eng. 70, $59-89$.

Belytschko, T., Liu, W. K., Moran, B., Elkhodary, K., 2013. Nonlinear Finite Elements for Continua and Structures, 2nd Edition. Wiley, Hoboken. Berger-Vergiat, L., McAuliffe, C., Waisman, H., 2014. Isogeometric analysis of shear bands. Comput. Mech. 54, 503-521.

Borja, R. I., 2008. Assumed enhanced strain and the extended finite element methods: A unification of concepts. Comput. Methods Appl. Mech. Eng. 197, 2789-2803.

Borja, R. I., Regueiro, R. A., 2001. Strain localization in frictional materials exhibiting displacement jumps. Comput. Methods Appl. Mech. Eng. $190,2555-2580$.

Bronkhorst, C. A., Cerreta, E. K., Xue, Q., Maudlin, P. J., Mason, T. A., Gray III, G. T., 2006. An experimental and numerial study of the localization behavior of tantalum and stainless steel. Int. J. Plast. 22, 1304-1335.

Bronkhorst, C. A., Gray III, G. T., Addessio, F. L., Livescu, V., Bourne, N. K., MacDonald, S. A., Withers, P. J., 2016. Response and representation of ductile damage under varying shock loading conditions in tantalum. J. Appl. Phys. 119, 085103.

Bronkhorst, C. A., Hansen, B. L., Cerreta, E. K., Bingert, J. F., 2007. Modeling the microstructural evolution of metallic polycrystalline materials under localization conditions. J. Mech. Phys. Solids 55, 2351-2383.

Cerreta, E. K., Bingert, J. F., Gray III, G. T., Trujillo, C. P., Lopez, M. F., Bronkhorst, C. A., Hansen, B. L., 2013. Microstructural examination of quasi-static and dynamic shear in high-purity iron. Int. J. Plast. 40, 23-38.

Cerreta, E. K., Frank, I. J., Gray III, G. T., Trujillo, C. P., Korzekwa, D. A., Dougherty, L. M., 2009. The influence of microstructure on the mechanical response of copper in shear. Mater. Sci. Eng. A 501, 207-219.

Das, A., Sivaprasad, S., Ghosh, M., Chakraborti, P. C., Tarafder, S., 2008. Morphologies and characteristics of deformation induced martensite during tensile deformation of $304 \mathrm{LN}$ stainless steel. Mater. Sci. Eng. A 486, 283-286.

Davies, C. H. J., 1997. Growth of nuclei in a cellular automaton simulation of recrystallisation. Scripta Mater. 36, 35-40.

Dodd, B., Bai, Y., 2012. Adiabatic Shear Localization: Frontiers and Advances, 2nd Edition. Elsevier, London.

Dodd, B., Bai, Y., 2014. Introduction to Adiabatic Shear Localization: Revised Edition. Imperial College Press, London.

Fish, J., Belytschko, T., 1988. Elements with embedded lozalization zones for large deformation problems. Comp. Struct. 30, $247-256$.

Follansbee, P. S., 2012. An internal state variable constitutive model for deformation of austenitic stainless steels. J. Eng. Mater. Technol. 134, 041007(1-10).

Follansbee, P. S., 2014. Fundamentals of Strength: Principles, Experiment, and Applications of an Internal State Variable Constitutive Formulation. Wiley-TMS, New York.

Follansbee, P. S., Huang, J. C., Gray III, G. T., 1990. Low-temperature and high-strain-rate deformation of nickel and nickel-carbon alloys and analysis of the constitutive behavior according to an internal state variable model. Acta Metall. Mater. 38, 1241-1254.

Follansbee, P. S., Kocks, U. F., 1988. A constitutive description of the deformation of copper based on the use of the mechanical threshold stress as an internal state variable. Acta Metall. 36, 81-93.

Garikipati, K., Hughes, T. J. R., 2000. A variational multiscale approach to strain localization-formulation for multidimensional problems. Comput. Methods Appl. Mech. Eng. 188, 39-60.

Hecker, S. S., Stout, M. G., Staudhammer, K. P., Smith, J. L., 1982. Effects of strain state and strain rate on deformation-induced transformation in 304 stainless steel: Part I. magnetic measurements and mechanical behavior. Metall. Trans. A 13, 619-626.

Hill, R., 1962. Acceleration waves in solids. J. Mech. Phys. Solids 10, 1-16.

Hines, J. A., Vecchio, K. S., 1997. Recrystallization kinetics within adiabatic shear bands. Acta Mat. 45, 635-649.

Huespe, A. E., Needleman, A., Oliver, J., Sanchez, P. J., 2009. A finite thickness band method for ductile fracture analysis. Int. J. Plast. 25, $2349-2365$. 
Huespe, A. E., Needleman, A., Oliver, J., Sanchez, P. J., 2012. A finite strain, finite band method for modeling ductile fracture. Int. J. Plast. 28, 53-69.

Hughes, T. J. R., 1980. Generalization of selective integration procedures to anisotropic and nonlinear media. Int. J. Numer. Methods Eng. 15, $1413-1418$.

Hughes, T. J. R., Winget, J., 1980. Finite rotation effects in numerical integration of rate constitutive equations arising in large-deformation analysis. Int. J. Numer. Methods Eng. 15, 1862-1867.

Jirásek, M., 2000. Comparative study on finite elements with embedded discontinuities. Comput. Methods Appl. Mech. Eng. 188, 307-330.

Kocks, U. F., Argon, A. S., Ashby, M. F., 1975. Thermodynamics and Kinetics of Slip. Vol. 19 of Progress in Materials Science. Pergamon, Oxford.

Leroy, Y., Ortiz, M., 1990. Finite element analysis of transient strain localization phenomena in frictional solids. Int. J. Numer. Analy. Methods Geomech. 14, 93-124.

Li, S., Liu, W.-K., Qian, D., Guduru, P. R., Rosakis, A. J., 2001. Dynamic shear band propagation and micro-structure of adiabatic shear band. Comput. Methods Appl. Mech. Eng. 191, 73-92.

Li, S., Liu, W.-K., Rosakis, A. J., Belytschko, T., Hao, W., 2002. Mesh-free Galerkin simulations of dynamic shear band propagation and failure mode transition. Int. J. Solids Struct. 39, 1213-1240.

Lin, Y. C., Chen, X.-M., 2011. A critical review of experimental results and constitutive descriptions for metals and alloys in hot working. Mater. Des. 32, 1733-1759.

Linder, C., Armero, F., 2007. Finite elements with embedded strong discontinuities for the modeling of failure in solids. Int. J. Numer. Methods Eng. 72, 1391-1433.

Ling, X., Belytschko, T., 2009. Thermal softening induced plastic instability in rate-dependent materials. J. Mech. Phys. Solids 57, 788-802.

McAuliffe, C., Waisman, H., 2013. Mesh insensitive formulation for initiation and growth of shear bands using mixed finite elements. Comput. Mech. 51, 807-823.

McAuliffe, C., Waisman, H., 2014. A Pian-Sumihara type element for modeling shear bands at finite deformation. Comput. Mech. 53, 925-940.

McVeigh, C., Liu, W. K., 2010. Multiresolution continuum modeling of micro-void assisted dynamic adiabatic shear band propagation. J. Mech. Phys. Solids 58, 187-205.

Medyanik, S. N., Liu, W. K., Li, S., 2007. On criteria for dynamic adiabatic shear band propagation. J. Mech. Phys. Solids 55, $1439-1461$.

Meyers, L. W., Manwaring, S., 1986. Critical adiabatic shear strength of low alloyed steel under compressive loading. In: Murr, L. E., Staudhammer, K. P., Meyers, M. A. (Eds.), Metallurgical Applications of Shock-Wave and High-Strain Rate Phenomena. Marcel Dekker, New York, Ch. 34, pp. 657-674.

Meyers, M. A., 1994. Dynamic Behavior of Materials. Wiley, New York, Ch. 15, pp. 448-487.

Meyers, M. A., Chen, Y.-J., Marquis, F. D. S., Kim, D. S., 1995. High-strain, high-strain-rate behavior of tantalum. Metall. Mater. Trans. A 26, 2493-2501.

Mourad, H. M., Bronkhorst, C. A., Addessio, F. L., Cady, C. M., Brown, D. W., Chen, S. R., Gray III, G. T., 2014. Incrementally objective implicit integration of hypoelastic-viscoplastic constitutive equations based on the mechanical threshold strength model. Comput. Mech. 53, 941-955.

Mourad, H. M., Garikipati, K., 2006. Advances in the numerical treatment of grain-boundary migration: Coupling with mass transport and mechanics. Comput. Methods Appl. Mech. Eng. 196, 595-607.

Okayasu, M., Fukui, H., Ohfuji, H., Shiraishi, T., 2013. Strain-induced martensite formation in austenitic stainless steel. J. Mat. Sci. 48, 6157-6166.

Oliver, J., Huespe, A., Sanchez, P., 2006. A comparative study on finite elements for capturing strong discontinuities: E-FEM vs X-FEM. Comput. Methods Appl. Mech. Eng. 195, 4732-4752.

Ortiz, M., Leroy, Y., Needleman, A., 1987. A finite element method for localized failure analysis. Comput. Methods Appl. Mech. Eng. 61, $189-214$.

Osovski, S., Rittel, D., Landau, P., Venkert, A., 2012. Microstructural effects on adiabatic shear band formation. Scripta Mater. 66, 9-12.

Osovski, S., Rittel, D., Venkert, A., 2013. The respective influence of microstructural and thermal softening on adiabatic shear localization. Mech. Mater. 56, 11-22.

Plohr, J. N., Plohr, B. J., 2016. Numerical simulation of systems of shear bands in ductile metal with inclusions. AIP Adv. 6, 025008.

Porter, D. A., Easterling, K. E., 1992. Phase Transformations in Metals and Alloys, 2nd Edition. Chapman \& Hall, London.

Preston, D. L., Tonks, D. L., Wallace, D. C., 2003. Model of plastic deformation for extreme loading conditions. J. Appl. Phys. 93, 211-220.

Rittel, D., Landau, P., Venkert, A., 2008. Dynamic recrystallization as a potential cause for adiabatic shear failure. Phys. Rev. Let. 101, 165501(4).

Rittel, D., Wang, Z. G., Merzer, M., 2006. Adiabatic shear failure and dynamic stored energy of cold work. Phys. Rev. Let. 96, 075502(4).

Simo, J. C., Hughes, T. J. R., 1986. On the variational foundations of assumed strain methods. J. Appl. Mech. 53, 51-54.

Simo, J. C., Hughes, T. J. R., 1998. Computational Inelasticity. Springer-Verlag, New York.

Song, J.-H., Areias, P., Belytschko, T., 2006. A method for dynamic crack and shear band propagation with phantom nodes. Int. J. Numer. Methods Eng. 67, 868-893.

Talonen, J., Hanninen, H., 2007. Formation of shear bands and strain-induced martensitie during plastic deformation of metastable austenitic stainless steels. Acta Mat. 55, 6108-6118.

Wang, B., Liu, Z., Wang, B., Zhao, S., Sun, J., 2014a. Microstructural evolution in adiabatic shear band in the ultrafine-grained austenitic stainless steel processed by multi-axial compression. Mater. Sci. Eng. A 611, 100-107.

Wang, B. F., Liu, Z. L., Wang, X. Y., Li, Z. Z., 2014b. An EBSD investigation on deformation-induced shear bands in a low nickel austenitic stainless steel under controlled shock-loading conditions. Mater. Sci. Eng. A 610, 301-308.

Wright, T. W., 2002. The Physics and Mathematics of Adiabatic Shear Bands. Cambridge University Press, Cambridge.

Wright, T. W., Batra, R. C., 1985. The initiation and growth of adiabatic shear bands. Int. J. Plast. 1, 205-212.

Wu, J.-Y., Li, F.-B., Xu, S.-L., 2015. Extended embedded finite elements with continuous displacement jumps for the modeling of localized failure in solids. Comput. Methods Appl. Mech. Eng. 285, 346-378.

Xu, Y. B., Zhong, W. L., Chen, Y. J., Shen, L. T., Liu, Q., Bai, Y. L., Meyers, M. A., 2001. Shear localization and recrystallization in dynamic deformation of 8090 Al-Li alloy. Mater. Sci. Eng. A 299, 287-295.

Xue, Q., Bingert, J. F., Henrie, B. L., Gray III, G. T., 2008. EBSD characterization of dynamic shear band regions in pre-shocked and as-received 304 strainless steels. Mater. Sci. Eng. A 473, 279-289. 
Xue, Q., Gray III, G. T., 2006. Development of adiabatic shear bands in annealed 3161 stainless steel: Part II. TEM studies of the evolution of microstructure during deformation localization. Metall. Mater. Trans. A 37, 2447-2458.

Xue, Q., Gray III, G. T., Henrie, B. L., Maloy, S. A., Chen, S. R., 2005. Influence of shock prestraining on the formation of shear localization in 304 stainless steel. Metall. Mater. Trans. A 36, 1471-1486.

Yang, Q., Mota, A., Ortiz, M., 2005. A class of variational strain-localization finite elements. Int. J. Numer. Methods Eng. 62, $1013-1037$. 